\title{
Thermal evolution of the core with a high thermal conductivity
}

\author{
Stéphane Labrosse* \\ Laboratoire de géologie de Lyon, ENS de Lyon, Université Lyon-1, Université de Lyon, 46, allée d'Italie, \\ 69007 Lyon, France.
}

\begin{abstract}
The rate at which heat is extracted across the core mantle boundary (CMB) is constrained by the requirement of dynamo action in the core. This constraint can be computed explicitly using the entropy balance of the core and depends on the thermal conductivity, whose value has been revised upwardly. A high order model (fourth degree polynomial of the radial position) for the core structure is derived and the implications for the core cooling rate and thermal evolution obtained, using the recent values of the thermal conductivity. For a thermal conductivity increasing with depth as proposed by some of these recent studies, a CMB heat flow equal to the isentropic value (13.25TW at present) leads to a $700 \mathrm{~km}$ thick layer at the top of the core where a downward convective heat flow is necessary to maintain an isentropic and well mixed average state. Considering a CMB heat flow larger than the well mixed isentropic value leads to an inner core less than $700 \mathrm{Myr}$ old and the thermal evolution of the core is largely constrained by the conditions for dynamo action without an inner core. Analytical calculations for that period show that a CMB temperature larger than $7000 \mathrm{~K}$ must have prevailed 4.5 Gyr ago if the geodynamo has been driven by thermal convection for that whole time. This raises questions regarding the onset of the geodynamo and its continuous operation for the last 3.5 Gyr. Implications regarding the evolution of a basal magma ocean are also considered.
\end{abstract}

Keywords: Core thermodynamics, Core evolution, Core mantle boundary heat flow, inner core age, early geodynamo

\section{Introduction}

The Earth magnetic field is generated by dynamo action in the liquid core, with motions thought to primarily result from convection. Since the original proposal of Braginsky (1963), the importance of compositional buoyancy from light element release at the inner core boundary (ICB) has been recognized. The contributions of thermal and compositional buoyancy have however changed during the evolution of the core, in particular with the onset of the inner core crystallization some time in the past. The rate at which the core evolves, reaches the freezing point at the center, and crystallizes to the present inner core size is controlled by the rate at which heat is extracted by mantle convection. This means that computing the thermal evolution of the core ultimately depends on our understanding of mantle convection and its evolution with time, a subject of active research. On the other hand, the study of the requirements to maintain a geodynamo for at least the last 3.45 Gyr (Tarduno et al., 2010) can lead to constraints on the core mantle boundary (CMB) heat flow, its evolution with time as well as the time evolution of the CMB temperature.

\footnotetext{
*Corresponding author

Email address: stephane.labrosse@ens-lyon.fr (Stéphane Labrosse)
} 
The link between the heat flow of across the CMB and the evolution of the core comes from the energy balance of the core. The link between this thermal evolution and the ability to maintain a geodynamo comes from the entropy balance. The theory behind these balances has been worked out in a number of studies (eg. Gubbins et al., 1979; Braginsky and Roberts, 1995; Lister and Buffett, 1995; Buffett et al., 1996; Labrosse et al., 1997; Labrosse, 2003; Gubbins et al., 2004; Nimmo, 2007) and the present paper builds on these results. In order to use the general theory to make a useable model for the thermal evolution of the core, expressions for the structure of the core, ie profiles of temperature, chemical potential, density, are needed. These can be obtained from classical thermodynamics relationships, provided some assumptions are made. The model developed here assumes the core to be isentropic and well mixed, owing to efficient convection. The reason to revisit this question comes mostly from the recent upward revision of the thermal conductivity of the core (de Koker et al., 2012; Pozzo et al., 2012; Gomi et al., 2013; Pozzo et al., 2014) which changes the amount of heat that is transferred by diffusion along the well mixed and isentropic temperature gradient, without any motion associated. This implies that, compared to the case with a lower conductivity, driving a dynamo with the same characteristics requires a higher CMB heat flow. Some of these questions have already been discussed by Pozzo et al. (2012) and Gomi et al. (2013) who also showed that stratified layers can exist in the core even if the CMB heat flow is large enough to drive a dynamo. Compared to the latter study, a higher order model for the structure of the core is derived here: the density is expressed as a fourth degree polynomial of radial position compared to the degree 2 used previously. Of course, all quantities that depend on density are computed analytically to the same order, in particular temperature. This proves necessary to resolve the apparent discrepancy between values of the well mixed and isentropic CMB heat flow given by Pozzo et al. (2012) and Gomi et al. (2013). Therefore the implications of the high thermal conductivity on the thermal evolution of the core need to be reconsidered.

The paper is structured as follows. Section 2 presents the high order self-consistent core structure and the values of the different parameters needed for the core evolution model. Section 3 discusses the energy and entropy balances of the core and shows how they can be used to get constraints on the $\mathrm{CMB}$ heat flow and core cooling rate at specific moments in its evolution where the thermal structure is well defined, the present time and the time just preceding the onset of the inner core crystallization. The age of the inner core is also discussed in section 3.4 and the possibility of core thermal stratification ins section 3.5. Then, section 4 shows how the energy and entropy balances can be used to compute a thermal evolution model with a minimal CMB heat flow. Implications are mostly discussed in section 5. Most expressions for the different terms in the energy and entropy equations are given explicitly in Appendix A.

\section{Radial structure of the core}

The low viscosity of the core (eg Poirier, 1988; de Wijs et al., 1998; Perrillat et al., 2010) makes convection currents very efficient at mixing all the extensive variables, and in particular entropy and composition. A good evidence of that efficiency comes from the observation of the secular variation of the magnetic field which implies a flow velocity of the order of $10^{-4} \mathrm{~m} \mathrm{~s}^{-1}$ (eg. Hulot et al., 2002), and implies, assuming a balance between the Coriolis and buoyancy forces, that the relative variation in density $\delta \rho / \rho \sim 10^{-9}$ (Braginsky and Roberts, 1995; Lister and Buffett, 1995; Labrosse et al., 1997). For this reason, we can write each quantity as the sum of a radial profile (subscript $a$ for average) and its lateral fluctuations (subscript $c$ for convective), $X=X_{a}+X_{c}$. The long term evolution is derived entirely from integrals involving the average profiles, convection being responsible for maintaining the core close to these profiles. This section is devoted to the derivation of a model for the radial structure of the core, based 
on the assumption that it is isentropic and well mixed. In the remainder of the paper, when a region of the core is stated as isentropic, it also means that it is compositionally well mixed. If stratification arises in a region of the core, either for thermal or composition reasons, the average profiles can differ from these profiles and evidence for such deviations exist both at the bottom (eg. Souriau and Poupinet, 1991; Song and Helmberger, 1995) and the top (eg. Tanaka, 2007; Helffrich and Kaneshima, 2010) of the core and will be discussed only at the end of the paper.

The assumption of a well mixed isentropic average state dictates the use of pressure $P$, specific entropy $s$ and mass fractions of light elements $\xi_{i}$ (one for each element considered) as state variables. The variation of each quantity $X_{a}$ with radius in the core can then be reduced to its pressure variation

$$
\frac{\partial X_{a}}{\partial r}=\left(\frac{\partial X}{\partial P}\right)_{s, \xi_{i}} \frac{\mathrm{d} P_{a}}{\mathrm{~d} r} .
$$

The partial derivative $\partial X / \partial P)_{s, \xi_{i}}$ is given by classical thermodynamics.

The average profiles of all the relevant quantities are obtained by solving a set of coupled differential equations that define this average state. Labrosse et al. (2001) showed how practical expressions for these profiles can be obtained and I follow the same approach here, but extend it to a higher order for reasons that will appear clearly at the outset. Pressure $P_{a}$, density $\rho_{a}$ and gravity $g_{a}$ are first considered as solution of the hydrostatic balance

$$
\frac{\mathrm{d} P_{a}}{\mathrm{~d} r}=-\rho_{a} g_{a}
$$

the Gauss theorem for gravity

$$
\frac{\mathrm{d}}{\mathrm{d} r}\left(r^{2} g_{a}\right)=4 \pi G \rho_{a} r^{2}
$$

and an equation of state

$$
\left(\frac{\partial \rho_{a}}{\partial P}\right)_{s, \xi_{i}}=\frac{\rho_{a}}{K_{s}}=\frac{\rho_{a}}{K_{0}+K_{0}^{\prime}\left(P-P_{0}\right)},
$$

$G$ being the gravitational constant. In equation (4), $K_{s}$ is the isentropic bulk modulus, defined by the first equality, and the second equality expresses the choice made here to consider only a linear variation with pressure, the subscript 0 giving values at the center $(r=0)$. This amounts to using the Adams-Williamson equation (Williamson and Adams, 1923), as is done for example in PREM to relate density to the seismic velocity (Poirier, 2000). This equation of state is simpler than the one used by Labrosse et al. (2001) but the results are compatible, at least to the second order that was considered in that former study.

Polynomial solutions are found for the set of equations (2)-(4) up to the desired order $N$ by simply expressing the density as $\rho_{a}=\sum_{0}^{N} a_{i} r^{i}$, and integrating in turn equation (3) to get the gravity to the same order, equation (2) to get the pressure and finally equation (4) to get the density. Comparison to the original form allows to identify the different coefficients and the result to order 4 is

$$
\begin{aligned}
\rho_{a} & =\rho_{0}\left(1-\frac{r^{2}}{L_{\rho}^{2}}-A_{\rho} \frac{r^{4}}{L_{\rho}^{4}}\right) \\
g_{a} & =\frac{4 \pi}{3} G \rho_{0} r\left(1-\frac{3}{5} \frac{r^{2}}{L_{\rho}^{2}}-\frac{3 A_{\rho}}{7} \frac{r^{4}}{L_{\rho}^{4}}\right), \\
P & =P_{0}-K_{0}\left(\frac{r^{2}}{L_{\rho}^{2}}+\frac{4 r^{4}}{5 L_{\rho}^{4}}\right)
\end{aligned}
$$


where

$$
L_{\rho}=\sqrt{\frac{3 K_{0}}{2 \pi G \rho_{0}^{2}}} ; \quad A_{\rho}=\frac{5 K_{0}^{\prime}-13}{10} .
$$

Note that the linear coefficient in the bulk modulus only appears at order 4 in $\rho_{a}$ and $g_{a}$, and that a quadratic term would only appear in the development to order 6 in radius. The density profile (5) does not include the density jump across the inner core boundary (ICB, radius $r_{I C}$ ), $\Delta \rho_{I C B}$. Assuming this density jump applies uniformly across the inner core, an additional gravity acceleration has to be included:

$$
\Delta g=\frac{4 \pi}{3} G \Delta \rho_{I C B}\left\{\begin{array}{ll}
r & \text { if } r \leq r_{I C} \\
\frac{r_{I C}^{3}}{r^{2}} & \text { if } r>r_{I C}
\end{array} .\right.
$$

This gives a pressure perturbation $\Delta P$ which can be obtained using the hydrostatic balance (2). This can be used to compute a density perturbation which, to leading order, is:

$$
\Delta \rho=-2 \Delta \rho_{I C B} \begin{cases}\frac{r^{2}}{L_{\rho}^{2}} & \text { if } r \leq r_{I C} \\ \frac{r_{I C}^{2}}{L_{\rho}^{2}}\left(2-\frac{r_{I C}}{r}\right) & \text { if } r>r_{I C} .\end{cases}
$$

The maximum value of this perturbation is found at the core mantle boundary ( $\mathrm{CMB}, r=r_{O C}$ ) where it amounts to $20 \%$ of the order 4 term in the density profile of equation (5). It is therefore neglected in the following.

\begin{tabular}{lll} 
Parameter & Order 2 & Order 4 \\
\hline$\rho_{0}\left(\mathrm{~kg} \mathrm{~m}^{-3}\right)$ & 12502 & 12451 \\
$L_{\rho}(\mathrm{km})$ & 7683 & 8039 \\
$A_{\rho}$ & - & 0.484 \\
$K_{0}(\mathrm{GPa})$ & 1291 & 1403 \\
$K_{0}^{\prime}$ & - & 3.567 \\
$\partial T_{a} / \partial r$ at $\mathrm{CMB}\left(\mathrm{K} \mathrm{km}^{-1}\right)$ & -0.89 & -0.97 \\
\hline
\end{tabular}

Table 1: Parameters for the polynomial least square fit of the density from PREM, for order 2 or 4 , as entering in equation (5) and implied values for $K_{0}, K_{0}^{\prime}$ and the isentropic temperature gradient at the CMB, computed using $T_{c}=5500 \mathrm{~K}$ as ICB temperature and $\gamma=1.5$ as Grüneisen parameter. The fit is done using only outer core density values from PREM with a $10 \mathrm{~km}$ sampling.

A density profile in the core can be determined from inversion of normal mode data (eg Masters and Gubbins, 2003) and PREM (Dziewonski and Anderson, 1981) provides such a profile as a polynomial fit. However, the polynomial for the outer core contains odd and even powers of the radius, up to power 3. Using this fit, a set of density values as function of radius can be predicted and then inverted back using equation (5). Results of the least square fit are given in table 1. No uncertainty can be obtained since none is available in PREM. PREM also provides values of $K_{s}$ and its fit in the outer core as function of pressure gives $K_{s}=187.35+3.3955 P$ (in GPa) which can be used to get $K_{0}=1417 \mathrm{GPa}$. These values are in reasonable agreement with those listed in table 1 and obtained from the density profile only. Note that the order 4 fit requires 1 less parameter than the order 3 fit given in PREM and the difference between densities predicted from the two expressions amounts to less that $0.05 \%$ in the whole outer core. The order 2 fit is also quite good with differences from PREM less than $0.35 \%$. 
Labrosse et al. (2001) used a different equation of state which resulted in an exponential form, compatible to second order with equation (5) but with a value of the density length scale of $7400 \mathrm{~km}$. Figure 1 shows the profiles obtained for these different choices, along with the PREM values. It shows that equation (5) gives the best fit, in particular when considering the gradient at the top of the core, which is important for the heat flow along the isentropic temperature profile, as is discussed now.

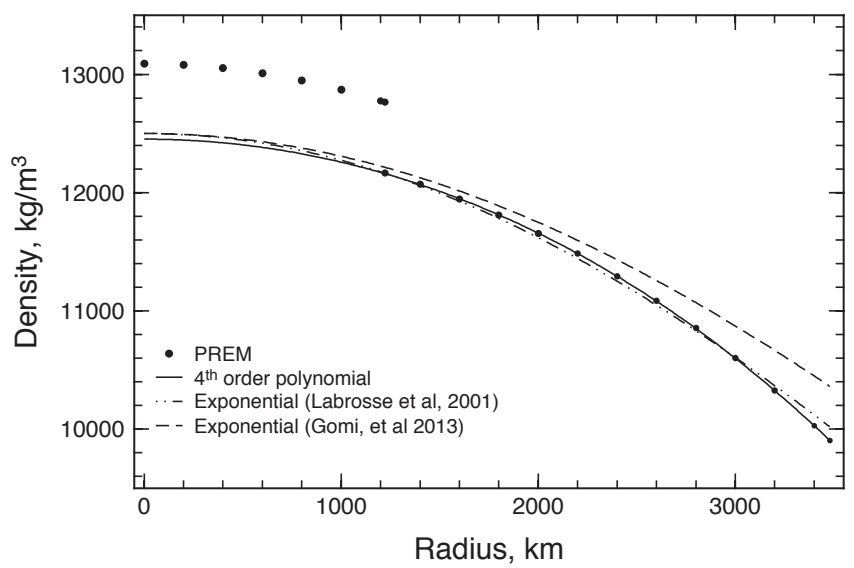

Figure 1: Density as function of radius in the core. Circles are the PREM values, the solid line is the $4^{\text {th }}$ order fit according to equation (5) with parameters given in table 2 . The dashed line and dash-dotted line are exponential forms $\rho=\rho_{0} \exp \left(-r^{2} / L^{2}\right)$ using $L=7765 \mathrm{~km}$ and $L=7400 \mathrm{~km}$, respectively.

The temperature profile, assumed isentropic, is obtained using (eg Poirier, 2000; Ricard, 2007; Hernlund and Labrosse, 2007)

$$
\left(\frac{\partial \ln T}{\partial \ln \rho}\right)_{s, \xi_{i}}=\gamma \Rightarrow T_{a}=T_{c}\left(\frac{\rho_{a}}{\rho_{c}}\right)^{\gamma},
$$

where the integration is performed assuming a constant Grüneisen parameter $\gamma$ (Alfè et al., 2001; Vočadlo et al., 2003) and $T_{c}$ and $\rho_{c}$ are the temperature and density at the ICB, respectively. Note that Ichikawa et al. (2014) find a value of the Grüneisen coefficient that decreases with depth in the core and is slightly smaller than the value of Alfè et al. (2001). The latter value is used nonetheless because it allows simpler calculations. The densities $\rho_{a}$ and $\rho_{c}$ are obtained from equation (5). Considering the different density models of figure 1 gives a variability of the temperature gradient at the top of the core of about $30 \%$, between -0.70 to $-0.97 \mathrm{~K} / \mathrm{km}$. This and different values of ICB temperature and core conductivity explain the difference of isentropic CMB heat flow between studies of Pozzo et al. (2012) and Gomi et al. (2013). Even the difference between order 2 and order 4 expression (table 1) is about $10 \%$ which justifies the development of the higher order theory.

Note that the density profile (5) is assumed not to vary with time, despite the inner core growth (as already discussed above) and the change of temperature. The temperature evolution with time comes in the model only through the change of the boundary condition, the central value or ICB liquidus. This is easily justified by inspection of equation (11): assuming a change of $T_{c}$ of order $1000 \mathrm{~K}$ gives a change of temperature anywhere in the core of about $20 \%$ directly from the effect of $T_{c}$ and of $\gamma \alpha \Delta T \simeq 1 \%$ from both changes of $\rho_{a}$ and $\rho_{c}$ that in fact largely compensate.

The temperature at the inner core boundary (ICB) is set to the liquidus temperature $T_{L}$ of the outer core material and depends on pressure and composition, both evolving with the 


\begin{tabular}{lll}
\hline Parameter & Notation & Value \\
\hline Core radius $^{a}$ & $r_{O C}$ & $3480 \mathrm{~km}$ \\
Present inner core radius $^{a}$ & $r_{I C p}$ & $1221 \mathrm{~km}$ \\
Thermal conductivity at the center $^{b}$ & $k_{0}$ & $163 \mathrm{~W} \mathrm{~m}^{-1} \mathrm{~K}^{-1}$ \\
Radial dependence of conductivity $^{b}$ & $A_{k}$ & 2.39 \\
Present inner core radius $^{b}$ & $r_{I C}(0)$ & $1221 \mathrm{~km}$ \\
Grüneisen coefficient $^{d}$ & $\gamma$ & 1.5 \\
Heat capacity $^{e}$ & $C_{P}$ & $750 \mathrm{JK}^{-1} \mathrm{~kg}^{-1}$ \\
Entropy of crystallisation $^{f}$ & $\Delta S$ & $127 \mathrm{JK}^{-1} \mathrm{~kg}^{-1}$ \\
Coefficient of compositional expansion $^{g}$ & $\beta$ & 0.83 \\
Compositional dependence of the liquidus temperature $^{h}$ & $\left(\frac{\partial T_{L}}{\partial \xi}\right)_{P}$ & $-2110^{3} \mathrm{~K}$ \\
Pressure dependence of the liquidus temperature $^{i}$ & $\left(\frac{\partial T_{L}}{\partial P}\right)_{\xi}$ & $9 \mathrm{~K} \mathrm{GPa}{ }^{-1}$ \\
Difference in mass fraction of light elements across ICB & $\xi_{f}$ & $5.6 \%$ \\
Present time liquidus at the ICB & $T_{L}\left(r_{I C}(0)\right)$ & $5500 \mathrm{~K}^{k}$ \\
Difference in mass fraction of light elements across ICB & $\Delta_{\xi} \rho$ & $580 \mathrm{~kg} \mathrm{~m}^{-3}$ \\
\hline
\end{tabular}

Table 2: Parameter values. ${ }^{a}$ from PREM (Dziewonski and Anderson, 1981). ${ }^{b}$ from Gomi et al. (2013). ${ }^{c}$ from PREM after subtraction of density jump across the ICB and a fit to equation (5). ${ }^{d}$ from Vočadlo et al. (2003). ${ }^{e}$ from Gubbins et al. (2003). ${ }^{f}$ from Hirose et al. (2013). ${ }^{g}$ computed using equation (17). ${ }^{h}$ from Alfè et al. (2007). ${ }^{i}$ from Alfè et al. (1999). ${ }^{j}$ combines effects from both O and S, computed by Labrosse (2014) from the equivalent in molar fraction given by Gubbins et al. (2013) in the case of the ICB density jump of Masters and Gubbins (2003). ${ }^{k}$ from Gubbins et al. (2013). ${ }^{l}$ from Masters and Gubbins (2003) and Alfè et al. (1999) (see text for discussion).

inner core growth. I assume a linear dependence on both mass fraction of light elements and pressure:

$$
T_{L}(P, \xi)=T_{L}\left(P_{0}, \xi_{0}\right)+\left(\frac{\partial T_{L}}{\partial P}\right)_{\xi}\left(P-P_{0}\right)+\sum_{i}\left(\frac{\partial T_{L}}{\partial \xi_{i}}\right)_{P}\left(\xi_{i}-\xi_{i 0}\right),
$$

$\xi_{i 0}$ being the mass fraction of light element $i$ in the core before inner core crystallization. The most likely light elements in the core are O, S and Si (Poirier, 1994) but their behaviors at the ICB are markedly different. Alfè et al. (2002) show that O has a very low partition coefficient, making it almost perfectly excluded upon inner core freezing, while $\mathrm{S}$ and Si have partition coefficients close to and undistinguishable from 1, respectively. This carries implications of two sorts. First, it implies that $\mathrm{O}$ is the most important element to drive compositional convection in the core, S playing a smaller role and Si none at all. Second, as discussed by Alfè et al. (2007), the effect of light elements on the liquidus temperature is proportional to the concentration difference between the solid and the liquid (for low concentrations) and therefore the liquidus at the ICB is influenced mostly by $\mathrm{O}$ and a little by $\mathrm{S}$.

The effect of light elements, both in driving core convection and decreasing the liquidus, depends therefore on their mass fraction difference across the ICB. Instead of writing the model for both $\mathrm{O}$ and $\mathrm{S}$, I keep only one fictitious element, with a mass fraction $\xi$, assume it is perfectly incompatible in the inner core and that its initial mass fraction is the sum of differences in mass fractions of $\mathrm{O}$ and $\mathrm{S}$ across the ICB: $\xi_{0}=\Delta \xi_{O}+\Delta \xi_{S}$. Therefore both elements contribute to the model while keeping it as simple as possible. The mass fraction $\xi$ in the outer core then 
increases during inner core growth as

$$
\xi(t)=\frac{\xi_{0} M_{C}}{M_{O C}(t)}=\xi_{0}\left(1+\frac{r_{I C}(t)^{3}}{L_{\rho}^{3} \mathrm{f}_{\mathrm{C}}\left(\frac{r_{O C}}{L_{\rho}}, 0\right)}+o\left(\frac{r_{I C}^{5}}{r_{O C}^{3} L_{\rho}^{2}}\right)\right)
$$

where $M_{C}$ and $M_{O C}$ are the total mass of the core and the time-evolving mass of the outer core, respectively. The function $\mathrm{f}_{\mathrm{C}}$ is an integral of the structure of the core used in most energy and entropy terms that is defined in Appendix A.1. The value of $\partial T_{L} / \partial \xi$ is taken from the equivalent given in molar fraction units by Alfè et al. (2007) and converted to mass fraction (see Labrosse, 2014, for details on this procedure).

The pressure effect comes from the pressure variation with radius as expressed by equation (7) which implies a variation of the pressure of freezing as the inner core grows and the additional effect due to increased density from inner core crystallization, the latter effect being negligible. Finally, the variation of the liquidus as function of the inner core radius reads as

$$
T_{L}\left(r_{I C}\right)=T_{L 0}-K_{0}\left(\frac{\partial T_{L}}{\partial P}\right)_{\xi} \frac{r_{I C}^{2}}{L_{\rho}^{2}}+\left(\frac{\partial T_{L}}{\partial \xi}\right)_{P} \frac{\xi_{0} r_{I C}^{3}}{L_{\rho}^{3} \mathrm{f}_{\mathrm{C}}\left(\frac{r_{O C}}{L_{\rho}}, 0\right)}
$$

with $T_{L 0}=T_{L}\left(P_{0}, \xi_{0}\right)$. Previously (Labrosse et al., 2001; Labrosse, 2003; Gomi et al., 2013), we used the Lindemann model of melting, which states $\partial \ln T_{L} / \partial \ln \rho=2(\gamma-1 / 3)$ and gives $\partial T_{L} / \partial P=2(\gamma-1 / 3) T_{L} / K_{0}$. Alternatively, Labrosse et al. (1997) and Pozzo et al. (2012) considered a constant value of $\partial T_{L} / \partial P$. We follow this latter approach here since this value is now constrained by ab initio calculations and experiments. Note however that the value from Alfè et al. (1999) that we use (see table 2 for a complete list of parameter values) is very similar to the value predicted by the Lindemann model, $9.15 \mathrm{~K} \mathrm{GPa}^{-1}$ using values from table 2 .

Before the existence of an inner core, the reference temperature and density in equation (11) are chosen as the central values, $T_{0}$ and $\rho_{0}$, simply giving

$$
T_{a}(r, t)=T_{0}(t)\left(1-\frac{r^{2}}{L_{\rho}^{2}}-A_{\rho} \frac{r^{4}}{L_{\rho}^{4}}\right)^{\gamma}
$$

The mass fraction $\xi$ of light elements is assumed uniform in the outer core, therefore neglecting any compositional stratification such as those proposed near the bottom (eg Souriau and Poupinet, 1991; Song and Helmberger, 1995) and the top of the core (eg Tanaka, 2007; Helffrich and Kaneshima, 2010). Since we consider only one light element, only one independent chemical potential is necessary to define the two components since the mass fractions add up to 1 and only the difference between the chemical potential of light element and that of Fe matters (e.g. Braginsky and Roberts, 1995, Appendix D). What is termed chemical potential in the following is precisely that difference.

The variation of chemical potential with radius is given by

$$
\frac{\partial \mu_{a}}{\partial r}=\left(\frac{\partial \mu}{\partial P}\right)_{s, \xi} \frac{\mathrm{d} P_{a}}{\mathrm{~d} r}=-\beta g_{a}
$$

$\beta$ being the coefficient of chemical expansion

$$
\beta \equiv-\frac{1}{\rho}\left(\frac{\partial \rho}{\partial \xi}\right)_{P, s}=\rho\left(\frac{\partial \mu}{\partial P}\right)_{s, \xi}
$$


(e.g. Lister and Buffett, 1995; Braginsky and Roberts, 1995, Appendix D). Assuming $\beta$ independent of radius, we get simply from equation (6) to the same order as previous expressions

$$
\mu_{a}(r, t)=\mu_{I C B}-\frac{2 \pi}{3} G \rho_{0} \beta\left(r^{2}-r_{I C}^{2}\right)\left(1-\frac{3}{10} \frac{r^{2}+r_{I C}^{2}}{L_{\rho}^{2}}\right) \equiv \mu_{I C B}+\mu^{\prime},
$$

$\mu_{I C B}$ being the chemical potential at the ICB across which it is continuous, an equilibrium between the solid and the liquid holding there. The exact value of $\mu_{I C B}$ will not enter the calculations and only the difference with the ICB value, $\mu^{\prime}$, will be used in computing the compositional energy. The value of $\beta$ can be estimated from the compositional contribution $\Delta_{\xi} \rho$ to the density jump across the ICB as (Lister and Buffett, 1995; Braginsky and Roberts, 1995):

$$
\beta=\frac{\Delta_{\xi} \rho}{\rho_{0} \xi} .
$$

$\Delta_{\xi} \rho$ can be evaluated by subtracting the density change due to phase transformation, $\Delta_{\phi} \rho=$ $220 \pm 20 \mathrm{~kg} \mathrm{~m}^{-3}$ (Alfè et al., 1999; Laio et al., 2000), to the total density change that is constrained by seismology. Unfortunately, large discrepancies exist between the different studies on the topic (Masters and Gubbins, 2003; Cao and Romanowicz, 2004; Koper and Dombrovskaya, 2005; Hirose et al., 2013, for a review) and the uncertainty in this key parameter directly affects the compositional energy. In this paper, I choose to use the value from Masters and Gubbins (2003) (see table 2) because it derives from a normal mode analysis and is therefore more likely to represent a global estimate, compared to wave reflection studies that can be influenced by the local interface state at the ICB. Additionally, it allows us to use consistent values for the concentrations in light elements, computed by Gubbins et al. (2013).

An important finding of the recent studies on the thermal conductivity of the core (de Koker et al., 2012; Pozzo et al., 2012; Gomi et al., 2013) is that it is much higher than previous estimates but also that it increases with depth in the core. Following Gomi et al. (2013), I will assume a quadratic variation with radius as

$$
k(r)=k_{0}\left(1-A_{k} \frac{r^{2}}{L_{\rho}^{2}}\right),
$$

the central value $k_{0}$ varying with temperature and composition, and $A_{k}$ a constant. The choice of parameterisation in equation (20) is made to give an expression similar to that of other parameters, notably density. The relationship to the expression used by Gomi et al. (2013) is straightforward. figure 2 shows profiles of the conductivity and heat flux along the isentrope at two periods with well defined thermal structures, the present and the time of the onset of the inner core. The composition effect on the thermal conductivity is important (Gomi et al., 2013) and, irrespective of the compositional model for the core, the fact that the inner core is depleted in light elements makes it more conductive (Pozzo et al., 2014). This is important when discussing the possibility of convection in the inner core (Labrosse, 2014) but since the cooling of the inner core is a small contribution in the global evolution of the core (eg. Labrosse et al., 2001), this issue needs not be discussed further here.

Even though several independent studies agree on the high value of thermal conductivity reported in table 2 (de Koker et al., 2012; Pozzo et al., 2012; Gomi et al., 2013), two other studies dispute it. Seagle et al. (2013), as Gomi et al. (2013), performed high pressure-ambient temperature electrical resistivity experiments and the experimental results agree with one another, within the error bounds. The papers differ in the proposed high temperature extrapolation 


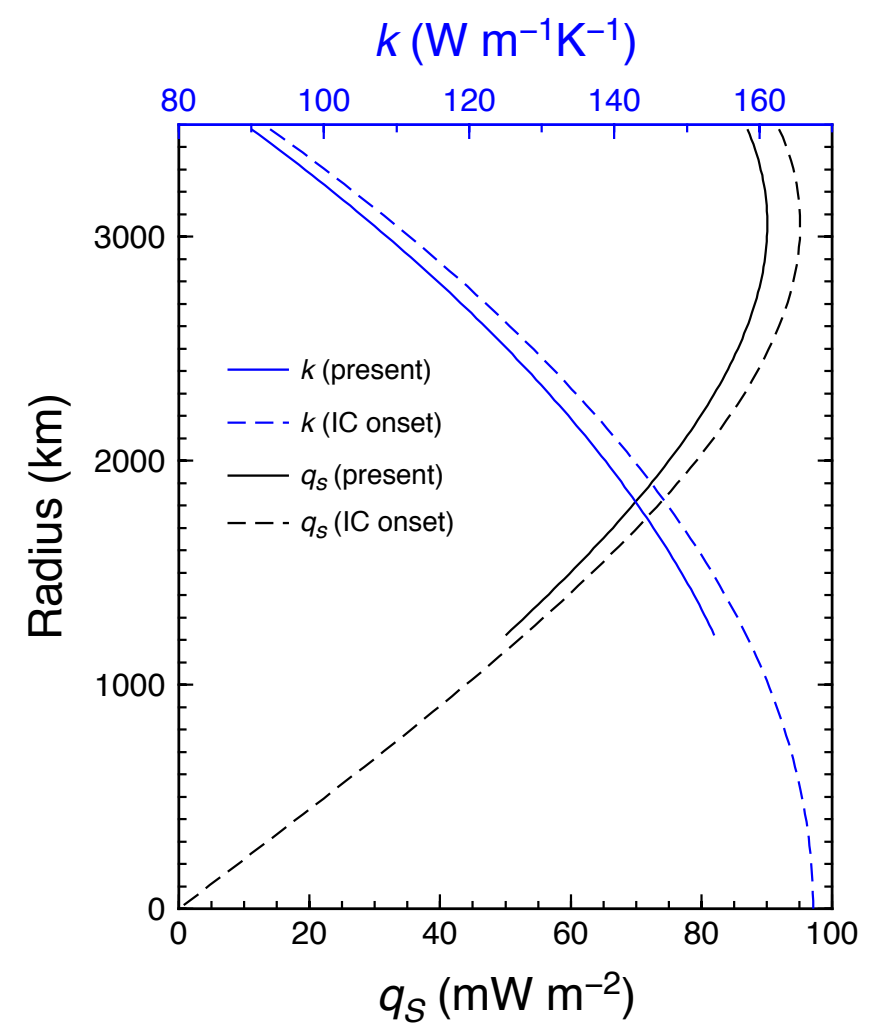

Figure 2: Profiles of thermal conductivity ( $k$, blue, top scale) and isentropic heat flux density $\left(q_{s} \equiv-k \partial_{r} T_{a}\right.$ black, bottom scale) as function of radial position in the core. Solid lines are for the present time while dashed lines are for the onset time of the inner core.

because Seagle et al. (2013) use the classical Bloch-Grüneisen formula all the way to the temperature of the core whereas Gomi et al. (2013) additionally take into account the saturation effect of resistivity of metals that is well documented for most metals (Gunnarsson et al., 2003) but not demonstrated at high pressure. However, the results of Gomi et al. (2013) agree well with the ab initio calculations of de Koker et al. (2012) and Pozzo et al. (2012). Zhang et al. (2015) used ab initio techniques to compute the contribution of electron-electron scattering to electrical resistivity of iron at core condition and found that, in contradiction to the assumption made by previous ab initio studies, it is as important as the electron-phonon scattering. Therefore they predict a thermal conductivity of the core similar to the value proposed by Seagle et al. (2013). However, recent high temperature measurements of electrical resistivity by Ohta et al. (2014) support the saturation model of Gomi et al. (2013) and casts doubts on the validity of these new ab initio calculations. It seems that the final word on this question has not yet been found and the possibility of a thermal conductivity in the range proposed by Seagle et al. (2013), $k=50 \mathrm{Wm}^{-1} \mathrm{~K}^{-1}$, will also be considered in the following.

\section{Energy and entropy balances}

\subsection{Global balances for the inner core era}

The general theory for the energy and entropy balance for the core has been worked out in several papers (eg. Gubbins et al., 1979; Braginsky and Roberts, 1995; Lister and Buffett, 1995; Labrosse et al., 1997; Labrosse, 2003; Gubbins et al., 2004; Nimmo, 2007) and will be only shortly recalled. The different terms in both balances are however derived with the core structure profiles discussed in section 2. This section covers the time since the inner core started to crystallize, the period before being discussed in the following section. 
The energy balance equation,

$$
Q_{C M B}=Q_{C}+Q_{I C B}+Q_{L}+E_{\chi}+Q_{R}
$$

simply states that the heat flow across the CMB, $Q_{C M B}$, is equal to the sum of five energy sources: secular cooling of the outer core, $Q_{C}$, heat flow from the inner core, $Q_{I C B}$ (equal to the secular cooling of the inner core plus radiogenic heating in the inner core), latent heat of freezing of the inner core, $Q_{L}$, compositional energy $E_{\chi}$ and possibly radiogenic heating $Q_{R}$, whose definitions are

$$
\begin{aligned}
Q_{C} & =-\int_{V_{O C}} \rho_{a} C_{p} \frac{\partial T_{a}}{\partial t} \mathrm{~d} V \\
Q_{I C B} & =4 \pi r_{I C}^{2} q\left(r_{I C}^{-}\right), \\
Q_{L} & =4 \pi r_{I C}^{2} \rho_{a}\left(r_{I C}\right) T_{L}\left(r_{I C}\right) \Delta S \frac{\mathrm{d} r_{I C}}{\mathrm{~d} t}, \\
E_{\chi} & =-\int_{V_{O C}} \rho_{a} \mu^{\prime} \frac{\partial \xi}{\partial t} \mathrm{~d} V, \\
Q_{R} & =h M_{O C}
\end{aligned}
$$

with $C_{p}$ the specific heat at constant pressure, $q\left(r_{I C}^{-}\right)$the heat flux density at the top of the inner core, $\Delta S$ the entropy of freezing, $h$ the radiogenic heating rate per unit mass, $V_{O C}$ the volume of the outer core.

The balance considered here does not include any contribution from chemical interaction with the mantle, the solute flux at the CMB being assumed null. The possibility of a solute flux in either direction is discussed in section 5 .

As discussed in many studies before (eg. Backus, 1975; Gubbins, 1977; Lister and Buffett, 1995; Braginsky and Roberts, 1995), the dissipation does not appear in this equation since it happens internally and is not lost from the core. It is in fact balanced internally by the work of buoyancy forces. This balance is classically obtained by summing the equations for the evolution of the kinetic energy (obtained from the scalar product of the momentum equation by the fluid velocity) and the magnetic energy (obtained from the scalar product of the induction equation with the magnetic field), using the anelastic approximation (Lister and Buffett, 1995; Braginsky and Roberts, 1995), to get the total dissipation $\Phi$ :

$$
\Phi \equiv \int_{V_{O C}} \boldsymbol{\tau}: \boldsymbol{\nabla} \boldsymbol{u} \mathrm{d} V+\int_{V_{\infty}} \frac{\boldsymbol{J}^{2}}{\sigma} \mathrm{d} V=\int_{V_{O C}} C \rho_{a} \boldsymbol{g}_{a} \cdot \boldsymbol{u} \mathrm{d} V-\partial_{t}\left(\int_{V_{O C}} \frac{\rho_{a} \boldsymbol{u}^{2}}{2} \mathrm{~d} V+\int_{V_{\infty}} \frac{\boldsymbol{B}^{2}}{2 \mu_{0}} \mathrm{~d} V\right)
$$

with $C=-\alpha_{s} s_{c}-\beta \xi$ the co-density introduced by Braginsky and Roberts (1995) to combine both thermal (entropic, $\alpha_{s}$ being the entropy expansion coefficient) and compositional buoyancy, $\boldsymbol{\tau}$ the stress tensor, $\boldsymbol{u}$ the fluid velocity, $\boldsymbol{J}$ the electric current density, $\sigma$ the electrical conductivity, $\mu_{0}$ the magnetic permeability and $V_{\infty}$ the volume of the whole space. This equation simply states that the power of the buoyancy force maintains viscous dissipation in the core, Ohmic dissipation in the whole space and allows changes of kinetic and magnetic energies. After averaging on a typical convective timescale, only secular changes of the kinetic and magnetic energies remain and these are clearly negligible (eg Gubbins, 1977). It is however important to note that the Ohmic dissipation term contains a contribution from regions outside the outer core, notably in the inner core and the mantle if it is not perfectly insulating. The geodynamo maintains a time varying magnetic field outside of the outer core (the corresponding magnetic energy being transported by the Poynting vector) which drives currents that contribute to the 
Ohmic dissipation. Denoting by $V_{e}$ the volume outside of the outer core $\left(V_{\infty}=V_{O C} \cup V_{e}\right)$, the energy balance (21) should therefore include on the right hand side a negative contribution of the form $-\int_{V_{e}} \boldsymbol{J}^{2} d V / \sigma$ (Braginsky and Roberts, 1995; Lister, 2003). Because of its very low electrical conductivity and therefore electric current, the mantle contribution can be safely neglected. The contribution of the inner core is also likely small, in particular because of the smallness of the inner core but also because the magnetic field only penetrates it by diffusion which reduces the importance of its small scales. Moreover, this heat production in the inner core is bound to escape it by diffusion or convection in the inner core and should therefore contribute positively to $Q_{I C B}$. Neglecting both the positive and negative contribution in the energy equation for the outer core is therefore likely to be a good approximation.

It appears already clearly that $Q_{L}$ is a function of $r_{I C}$ and is proportional to its growth rate but it is also the case for $Q_{C}$ and $E_{\chi}$, because the variation of both $\xi$ and $T_{a}$ are linked to the inner core growth. Each of these terms, say $X$, can then be written

$$
Q_{X}=P_{X}\left(r_{I C}\right) \frac{\mathrm{d} r_{I C}}{\mathrm{~d} t}
$$

The expressions for the different $P_{X}$ are obtained directly from the definitions of the different $Q_{X}$ and integrations of the basic profiles discussed in section 2. In some cases, the integration is analytic and in some others a polynomial expansion is used, to the same order as before. Detailed expressions are given in the appendix ( $\S$ Appendix A).

The radiogenic heating term is readily computed for any assumed concentration of radioactive elements and, assuming equal partition of radioactive elements between the inner core and the outer core, depends on time through the change of radiogenic heating and the change of outer core mass from inner core growth. If the partition coefficient for radioactive elements at the ICB is different from 1, it should also be included in the calculation, making the value of $h$ change not only from the decay but also from partitioning. However, the resulting effect should be minor since the inner core is too small for a strong partitioning to affect the global balance of the core. In the following we will only consider the possibility of potassium, since it is the radioactive element most often proposed to be in the core. Uranium and thorium have also been discussed (Labrosse et al., 2001; Wheeler et al., 2006; Malavergne et al., 2007; Bouhifd et al., 2013) but their contribution has been considered too small to be discussed further here.

The heat flow at the inner core boundary is computed from a numerical solution of conduction in the inner core taking into account its growth rate (Labrosse, 2014). It is however a small player and no large error can be made by either assuming an infinite conductivity (and therefore a uniform temperature) or zero conductivity (and therefore a null heat flow) in the inner core (Buffett et al., 1992). A reasonable intermediate estimate (Labrosse et al., 2001) comes from assuming the temperature to follow the same isentrope as in the outer core, which allows us to include the heat flow across the ICB into the cooling term, by performing the integration on the right hand side of equation (22) over the whole core. This is the option chosen to compute the age of the inner core $(\S 3.4)$.

The entropy balance equation,

$$
\frac{Q_{C M B}}{T_{C M B}}=\frac{Q_{I C B}+Q_{L}}{T_{I C B}}+S_{C}+S_{R}+S_{k}+S_{\phi},
$$

states that the outflow of entropy at the CMB is balanced by the sum of the inflow at the ICB (from the heat flow across the ICB and the latent heat), the change of entropy content from cooling, $S_{C}$, the entropy production from radioactivity, $S_{R}$, heat conduction, $S_{k}$, and Ohmic and viscous dissipation $S_{\phi}$. The four last source terms on the right hand side of equation (29) 
are defined as:

$$
\begin{aligned}
S_{C} & =-\int_{V_{O C}} \frac{\rho_{a} C_{p}}{T_{a}} \frac{\partial T_{a}}{\partial t} \mathrm{~d} V, \\
S_{R} & =\int_{V_{O C}} \frac{\rho_{a} h}{T_{a}} \mathrm{~d} V, \\
S_{k} & =\int_{V_{O C}} k\left(\frac{\nabla T_{a}}{T_{a}}\right)^{2} \mathrm{~d} V \\
S_{\phi} & =\int_{V_{O C}}\left(\frac{\boldsymbol{\tau}: \nabla \boldsymbol{u}}{T_{a}}+\frac{\boldsymbol{J}^{2}}{\sigma T_{a}}\right) \mathrm{d} V .
\end{aligned}
$$

Each of these term is the integral of a heat source divided by the temperature at which it is provided. An effective temperature $T_{X}$ can then be defined for each term as

$$
S_{X}=\frac{Q_{X}}{T_{X}} .
$$

Combining the entropy equation (29) with that for the conservation of energy (21) allows to write an efficiency equation that relates the total dissipation $\Phi$ to the different energy sources:

$$
\begin{aligned}
\Phi= & \frac{T_{\Phi}\left(T_{C}-T_{C M B}\right)}{T_{C M B} T_{C}} Q_{C}+\frac{T_{\Phi}\left(T_{I C B}-T_{C M B}\right)}{T_{C M B} T_{I C B}}\left(Q_{I C B}+Q_{L}\right) \\
& +\frac{T_{\Phi}\left(T_{R}-T_{C M B}\right)}{T_{C M B} T_{R}} Q_{R}+\frac{T_{\Phi}}{T_{C M B}} E_{\chi}-T_{\Phi} S_{k} .
\end{aligned}
$$

This equation makes clear what are the efficiency factors for converting any energy source in the core into dissipation, that is work (eq. 27). Heat sources provided at a temperature $T_{X}$ are impeded by a Carnot like factor of the form $\left(T_{X}-T_{C M B}\right) / T_{X}$, although the complete conversion factors are larger than the classical Carnot ones by a factor $T_{\Phi} / T_{C M B}$ because dissipation occurs inside the core and is not lost from the system, opposite to what happens in classical Carnot engines. The existence of the Carnot efficiency factors implies that driving the geodynamo by lateral variations of CMB heat flux is highly ineffective: the lateral temperature differences at the top of the core make the Carnot factor of the order $10^{-9}$. The compositional energy on the other hand has a much larger conversion factor, $T_{\Phi} / T_{C M B}$, because it is not a heat source and therefore does not contribute to the entropy budget (eq. 29). Finally, equation (35) also shows that the dissipation by thermal diffusion, $S_{k}$, is a sink that has to be exceeded for any convection and dynamo action to happen.

Calculation of the different conversion factors (hereafter denoted by $\eta_{X}$ for energy source $X)$ requires knowledge of the temperature $T_{\Phi}$ at which dissipation occurs in average, which is not known a priori. However, it is bounded by the temperatures at the ICB and the CMB, which gives a maximum range of the factor $T_{\Phi} / T_{C M B}$ between 1 and 1.36 . This could appear like a large uncertainty ( $\pm 15.3 \%$ when considering the median value) but it should be realized that the extreme values could only be obtained if all the dissipation occurs either at the ICB (for the largest factor) or at the CMB (for the lowest), which is quite unlikely. In other words, the actual uncertainty on the value of this factor is much lower, although not known. The possibility of stably stratified layers, discussed below, is likely to affect the distribution of the dissipation which should happen mostly in regions that are strongly driven. This question should be adressed with fluid dynamics models of the core. In the following, I will assume that 
dissipation is evenly distributed in the core so that

$$
T_{\Phi}=\left(\frac{1}{M_{O C}} \int_{V_{O C}} \frac{\rho_{a} \mathrm{~d} V}{T_{a}}\right)^{-1}
$$

An explicit derivation of this temperature as well as $T_{C}$ and $S_{k}$ is provided in Appendix A.

All terms on the right hand side of equation (35), save the radiogenic one and that from conduction, can obviously be written as functions of the inner core radius multiplied by its growth rate. Two strategies are possible. One can use an assumed CMB heat flow to obtain the present inner core growth rate from the energy equation (21) and then use the dissipation equation (35) to compute the dissipation. Alternatively, one can use constraints on the dissipation to get the growth rate of the inner core from the dissipation equation and then predict the CMB heat flow using the energy equation. Both strategies have their merits and limits, in particular the fact that both the present CMB heat flow (Lay et al., 2008) and Ohmic dissipation (Christensen, 2010) are poorly known, and the situation is of course worse for past periods. However, an important constraint on the total dissipation is that it must be strictly positive and, with the large values proposed recently for the thermal conductivity, this constraint is already quite strong, as shown in section 3.3.

\subsection{Global balances before the inner core}

Before the onset of inner core crystallization, the energy balance equation is simply

$$
Q_{C M B}=Q_{C}+Q_{R}
$$

with

$$
\begin{aligned}
& Q_{C}=-\int_{V_{C}} \rho_{a} C_{p} \frac{\partial T_{a}}{\partial t} \mathrm{~d} V=-\frac{4 \pi}{3} \rho_{0} C_{p} L_{\rho}^{3} \mathrm{f}_{\mathrm{C}}\left(\frac{r_{O C}}{L_{\rho}}, \gamma\right) \frac{\mathrm{d} T_{0}}{\mathrm{~d} t}, \\
& Q_{R}=M_{C} h(t)=\frac{4 \pi}{3} \rho_{0} L_{\rho}^{3} \mathrm{f}_{\mathrm{C}}\left(\frac{r_{O C}}{L_{\rho}}, 0\right) h(t),
\end{aligned}
$$

$V_{C}$ and $M_{C}$ being the volume and mass of the whole core and the function $\mathrm{f}_{\mathrm{C}}$ being given in Appendix A.3. Similarly to the case with a growing inner core, the entropy balance can be combined with the energy balance to get an efficiency equation:

$$
\Phi=\frac{T_{\Phi}\left(T_{C}-T_{C M B}\right)}{T_{C M B} T_{C}} Q_{C}+\frac{T_{\Phi}\left(T_{R}-T_{C M B}\right)}{T_{C M B} T_{R}} Q_{R}-T_{\Phi} S_{k}
$$

The expressions for $T_{\Phi}, T_{R}$ and $S_{k}$ are the same as before and obtained by setting $r_{I C}=0$.

As for the period since the onset of inner core crystallization, the combined use of the energy and dissipation equations can constrain the CMB heat flow and core cooling rate. This is the subject of the next section.

\subsection{Efficiency factors}

The first application of the model discussed above is to get constraints on the CMB heat flow at different times, and in particular at the present time and just before the onset of the inner core crystallization since these are two periods at which the thermal structure is well defined. Figure 3A shows the rate of core cooling just before the onset of the inner core crystallization needed to maintain a total dissipation between 0 and 3 TW either without any radioactive 

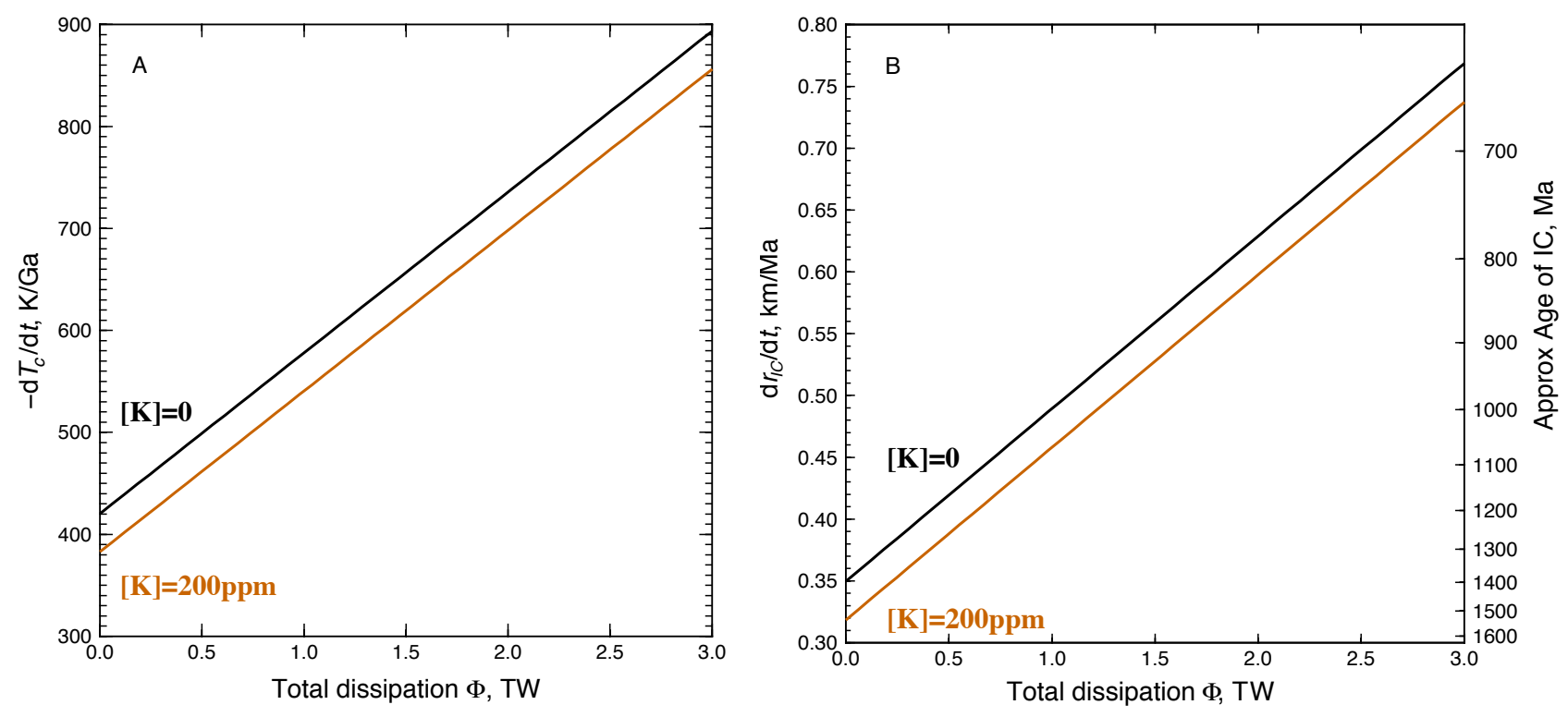

Figure 3: Core cooling rate just before the onset of inner core crystallization (A) and present inner core growth rate (B) as function of total dissipation. Also given on the right axis of $\mathrm{B}$ is the approximate age of the inner core, in Myr. See $\S 3.4$ for details of this calculation.

heating or with a radiogenic heating coming from a concentration of potassium of 200ppm, which is probably an overestimate of the amount present in the core (Hirose et al., 2013; Nomura and Hirose, 2014). This figure shows that even to maintain a null dissipation requires a core cooling rate of $382 \mathrm{~K} / \mathrm{Gyr}$ and $420 \mathrm{~K} / \mathrm{Gyr}$ with and without radioactivity, respectively. The age of the inner core being rather small, as discussed below, simple extrapolation of such a cooling rate easily produce a total cooling of the core in excess of $1000 \mathrm{~K}$ since its formation, if a magnetic field is to be maintained all along with a fully isentropic core.

The reason for the moderate effect of radioactivity can be understood by looking at the different contributions to the total dissipation and the CMB heat flow as function of the target dissipation, with and without radioactivity (fig. 4). Without radioactivity, one can see that an exactly null dissipation is obtained when the contribution from the secular cooling is exactly balanced by the dissipation due to conduction along the isentrope but that it requires a CMB heat flow greater than the isentropic one, by about $2 \mathrm{TW}$. This is due to the increase of the thermal conductivity with depth, which can cause the deepest core to be thermally stratified if the CMB heat flow is not large enough, or the whole core if it is lower or equal to the isentropic value. The situation with an exactly null dissipation is obtained when the excess buoyancy available in the upper part of the core can, at least in terms of global thermodynamic balances, maintain the isentropic temperature gradient in the whole core. This issue is discussed further in section 3.5. Including 200ppm of potassium produces about $2 \mathrm{TW}$ at the period just before the inner core crystallization which amounts to about $12 \%$ of the minimum secular cooling without radioactivity. But since the radiogenic heating has an efficiency in the conversion to dissipation similar but smaller than that of secular cooling, a slightly larger CMB heat flow is necessary for the same dissipation and the cooling rate is decreased by only $8 \%$.

Let us now discuss the situation for the present time. Figure $3 \mathrm{~B}$ shows the present inner core (IC) growth rate (left axis) and the corresponding inner core age $\left(a_{I C}\right.$, right axis) for a given dissipation between 0 and $3 \mathrm{TW}$, with and without radioactivity. The correspondance 

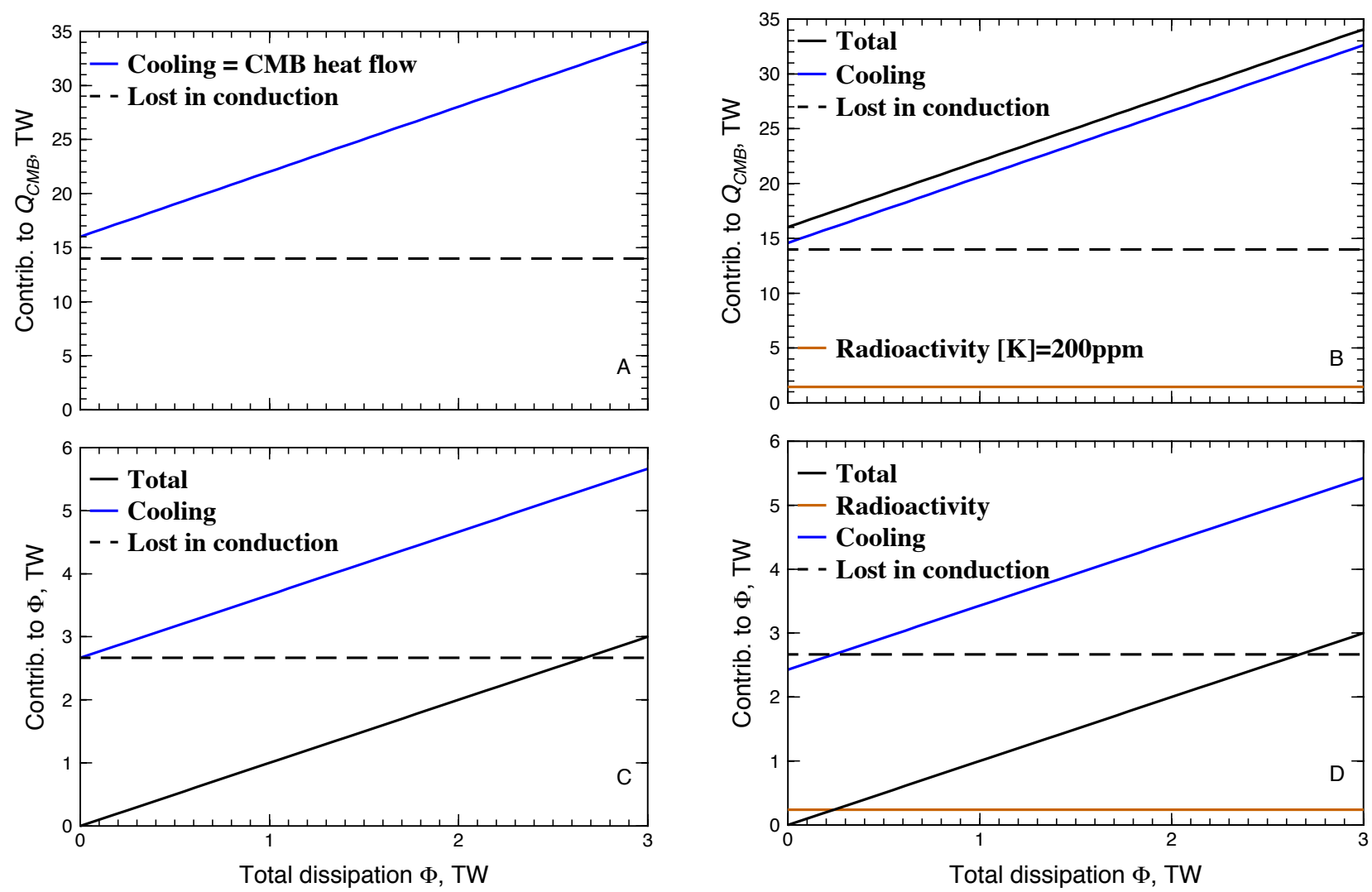

Figure 4: Contributions to the core energy balance (A and B) and total dissipation (C and D) as a function of the wanted total dissipation for the period just preceding the onset of the inner core crystallization, without any radioactivity (A and $\mathrm{C}$ ) or including $200 \mathrm{ppm}$ of potassium (B and D).

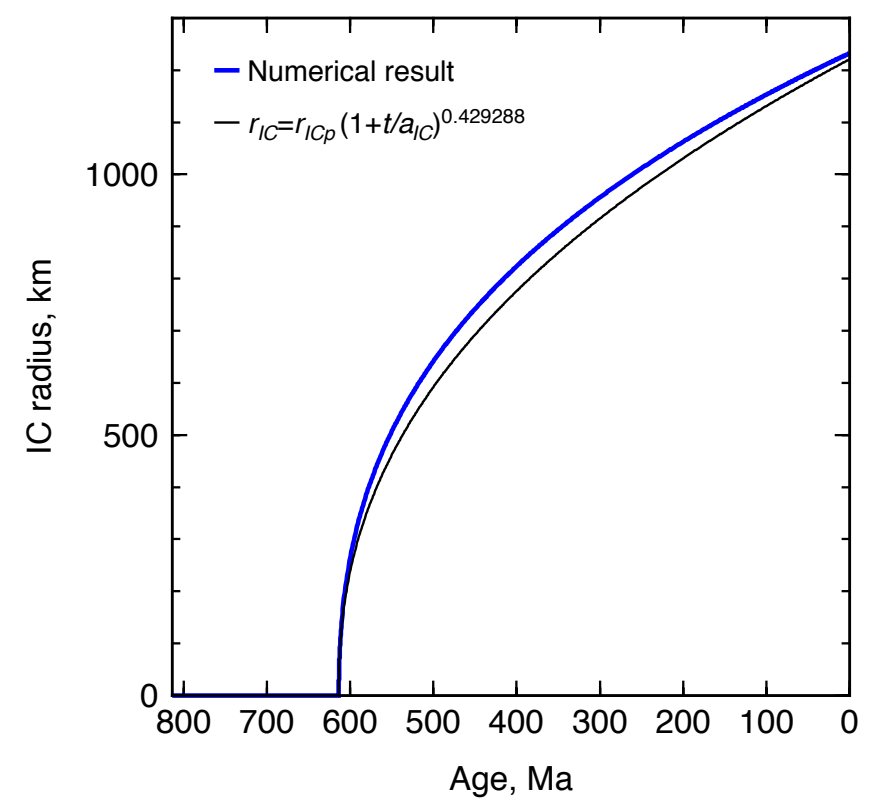

Figure 5: Typical example of inner core growth curve showing that it follows a power law with an exponent close to 0.4 . 
between present IC growth rate and age is computed assuming that the inner core grows as

$$
r_{I C}(t)=r_{I C p}\left(\frac{t}{a_{I C}}+1\right)^{0.4},
$$

$t=0$ being the present time, with $r_{I C p}$ the present inner core radius. The value of the exponent is representative of values found by fitting numerical results of the termal evolution models discussed later. Figure 5 shows a case with the exponent equal to 0.43 but different values around 0.4 are obtained depending on the CMB heat flow history.

As for the cooling rate before the inner core, the present growth rate of the inner core is not strongly influenced by the presence of $200 \mathrm{ppm}$ of potassium and the reason is the same: the low efficiency of conversion of radiogenic heating into dissipation. This can be seen on figure 6 which shows the different contributions to the dissipation and the CMB heat flow, for the present core thermal structure. The much larger efficiency of compositional energy over all heat sources, as apparent in equation (35), can be seen here in the fact that it contributes little to the energy budget (fig. 6A and B) but it is the major contributor to the dissipation (fig. 6C and D). With $200 \mathrm{ppm}$ of potassium, radiogenic heating is a small contributor to the energy budget, even for the minimum dissipation, and an even smaller contributor to the total dissipation. Note that the minimum requirement, a null dissipation, gives a CMB heat flow of about 7 TW without radioactivity and almost 8 TW with 200ppm of potassium, so that since the onset of the inner core crystallization, radiogenic heating cannot be considered as helping the dynamo. The reason for this is simple: including radioactivity decreases the cooling of the core and therefore the flux of light elements at the ICB and the associated compositional energy. Since that energy is precisely the most efficient one, this leads to a decrease of the overall efficiency and cranks up the demand on the CMB heat flow. The minimum requirements for the dynamo, the null total dissipation, gives an approximate age of the inner core between 1.40 and 1.53 Gyr (fig. 3B) depending on the presence of radioactivity or not. This must therefore be considered as the maximum age of the inner core.

Compared to the situation before the existence of the inner core, the null dissipation gives a value for the CMB heat flow much lower than the isentropic one. This is possible because of the high efficiency of compositional energy that can in that case compensate for the thermally stabilizing effect of having a subisentropic CMB heat flow. Whether it is dynamically possible and geophysically acceptable will be discussed in section 3.5 but it is at least acceptable from a purely thermodynamics point of view.

\subsection{Age of the inner core}

\begin{tabular}{lcr}
\hline Energy & Symbol & Value $\left(10^{28} \mathrm{~J}\right)$ \\
\hline Latent & $\mathcal{L}$ & 6.88 \\
Compositional & $\mathcal{X}$ & 4.69 \\
Cooling & $\mathcal{C}$ & 18.13 \\
\hline Total & & 29.7 \\
\hline
\end{tabular}

Table 3: Total energies

As shown by Labrosse et al. (2001), the energy balance equation (21) can be integrated between the onset time of inner core crystallisation $\left(t=-a_{I C}\right)$ and the present $(t=0)$ to yield an equation for the age of the inner core:

$$
\int_{-a_{I C}}^{0} Q_{C M B}(t) \mathrm{d} t=\mathcal{C}+\mathcal{L}+\mathcal{X}+M_{C} \int_{-a_{I C}}^{0} h(t) \mathrm{d} t
$$



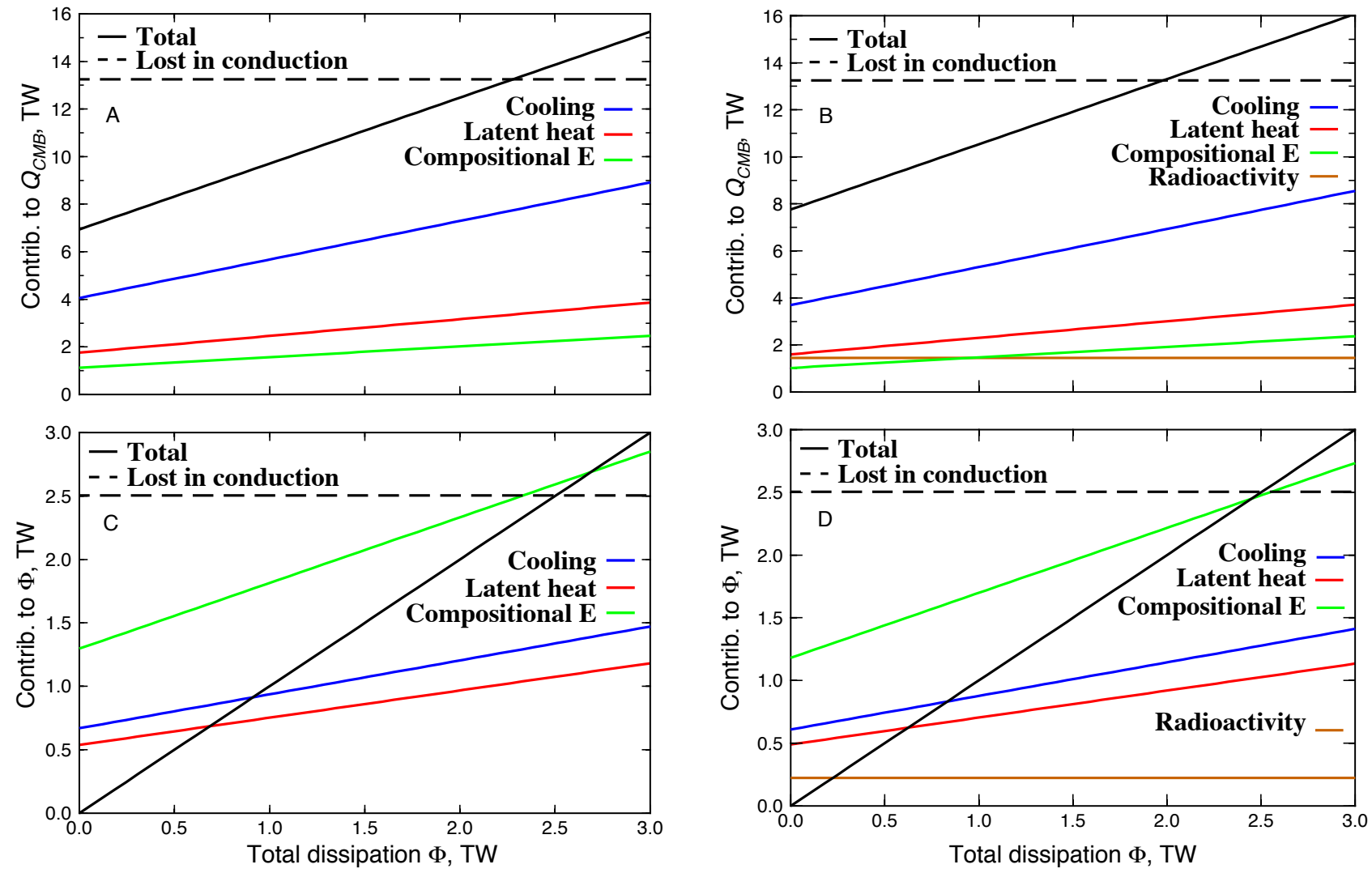

Figure 6: Contributions to the core energy balance (A and B) and dissipation (C and D) as a function of the wanted total dissipation for the present period, with inner core crystallization, without any radioactivity (A and C) or including 200 ppm of potassium (B and D). 
with

$$
\begin{aligned}
\mathcal{C} & =\int_{0}^{r_{I C p}} \tilde{\mathrm{P}}_{\mathrm{C}}(u) \mathrm{d} u, \\
\mathcal{L} & =\int_{0}^{r_{I C p}} \mathrm{P}_{\mathrm{L}}(u) \mathrm{d} u, \\
\mathcal{X} & =\int_{0}^{r_{I C p}} \mathrm{P}_{\chi}(u) \mathrm{d} u .
\end{aligned}
$$

The secular cooling term (eq. 43) includes the inner core, assuming the inner core temperature follows the same isentrope as that of the outer core, which is a good approximation in this calculation (Labrosse et al., 2001). See appendix Appendix A for the detailed expression of the different $\mathrm{P}$ functions and the resulting total energies $\mathcal{C}, \mathcal{L}$ and $\mathcal{X}$.

It may seem that the radiogenic heating term on the right hand side of equation (42) assumes that the concentration in radioactive elements is uniformly distributed in the core since $M_{C}$ is its total mass. However, even if crystallisation of the inner core fractionate the concentration of heat producing elements, the total heat production is conserved and the expression is exact, as long as $h(t)$ is understood as the average heat production rate in the core. The time variation obviously comes from radioactive decay but could also include chemical exchange with the mantle if one thinks such a possibility is important. As discussed above, the recent high estimates of the thermal conductivity of the core brings requirements on the CMB heat flow to so high values that the acceptable range of potassium concentration provides a quite small contribution to the balance. This will be discussed further below.

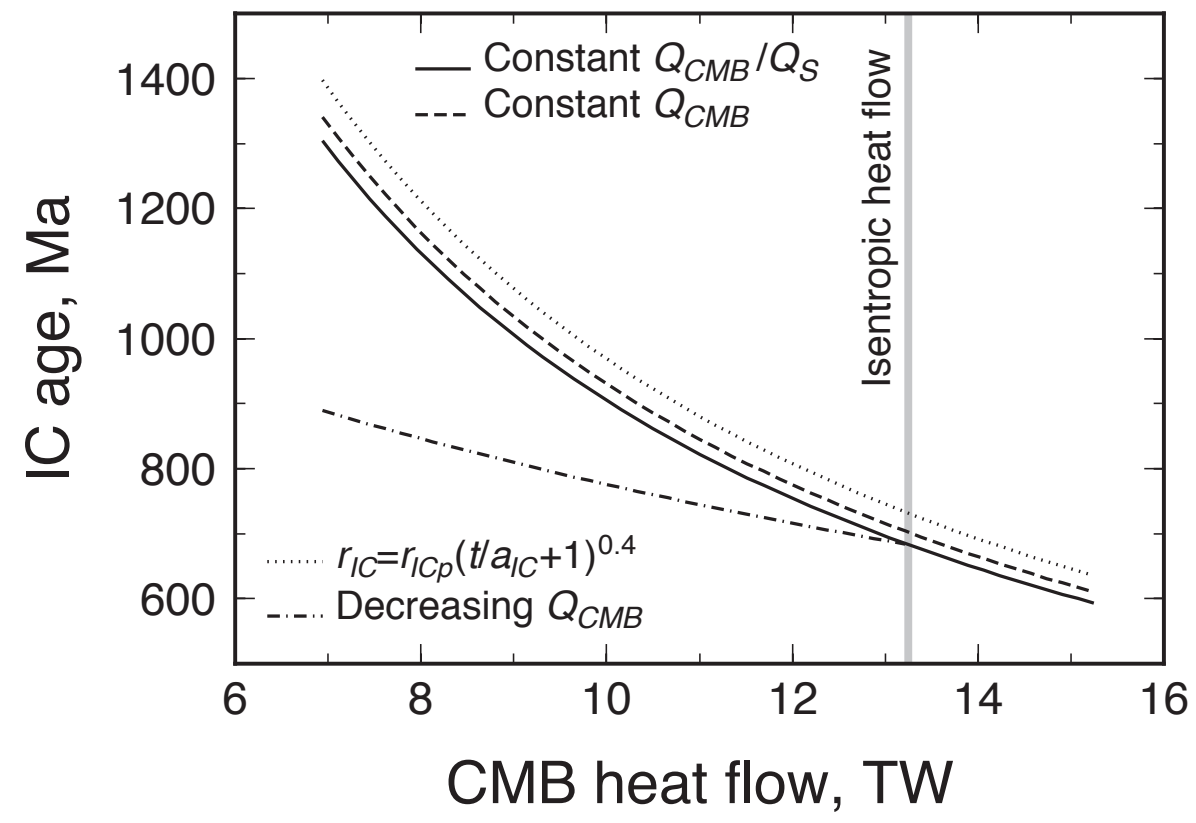

Figure 7: Age of the inner core as function of the present CMB heat flow. The solid line is obtained assuming a linear evolution of the CMB heat flow keeping the same ratio to the isentropic value while the dashed line assumes a constant CMB heat flow. The dotted line is obtained from just the present inner core growth rate and assuming a power law growth history. The dash-dotted line is obtained assuming a linearly decreasing CMB heat flow between the isentropic value at the onset of the inner core and the present value given in abscissa. The vertical grey line gives the present time value of the isentropic heat flow at the CMB. See text for details.

Table 3 gives the values of the total energies computed using the parameters of table 2 . These energies are independent of any choice of CMB heat flow but to get the age of the inner 
core from these numbers one needs to integrate the CMB heat flow over time. Using a constant CMB heat flow leads to a straightforward integration and a direct estimate of the age of the inner core. Alternatively, one can assume a CMB heat flow varying linearly or exponentially with time (Labrosse et al., 2001) but these should just be considered as example calculations since the heat flow at the CMB is controlled by mantle convection and has no reason to evolve monotonically (eg. Nakagawa and Tackley, 2010). In order to estimate the range of possible inner core ages, I consider three options: (1) a constant CMB heat flow, (2) a linearly varying one, with the present time value and the value at the onset of the inner core crystallization having the same ratio to the isentropic heat flow at each of the corresponding times, and (3) a linearly varying one between the isentropic value at the onset of the inner core crystallization and an arbitrary present value. In all three cases, the age of the inner core can be computed analytically from the total energy and the present value of the CMB heat flow. The result of these calculations is presented on figure 7. In addition, the age of the inner core is also computed in the same way as for figure 3, that is assuming a power law growth history of the inner core, with an exponent equal to 0.4, and the present growth rate computed from the energy balance. The range of present CMB heat flows is the one obtained in section 3.3 and correspond to a present dissipation in the range $[0-3] \mathrm{TW}$. Therefore, the maximum value obtained is a real maximum and corresponds to a null dissipation at present.

The first two estimates give similar results, because the isentropic value does not change greatly between the onset of the inner core and the present time. The approximate value obtained assuming a power law growth history also give a similar value, which shows that it is a good approximation to the case of nearly constant CMB heat flow. However, these scenarios of a nearly constant CMB heat flow are quite unlikely when the value considered is lower than the isentropic one because the heat flow must have been super-isentropic before the existence of the inner core for dynamo action to have been possible and it would require a drop of the CMB heat flow at the onset time. But since the CMB heat flow is controlled by mantle convection with little retroaction from the core, such a drop would be only fortuitous and therefore unlikely. A more realistic lower bound for the $\mathrm{CMB}$ heat flow, therefore a higher bound on the age of the inner core, is obtained for a CMB heat flow exactly equal to the isentropic value at the onset of the inner core and a present value similar to that chosen before. The result of such a calculation is shown on figure 7 as a dash-dotted line and shows a maximum age of about 900 Myr. In the next section, I will show that the present CMB heat flow is unlikely to be lower than the isentropic value and this implies an inner core age lower than 700 Ma.

\subsection{Subshell balances and thermal stratification}

Gomi et al. (2013) showed how the energy balance can be integrated in a sub-shell in the core in order to compute the convective heat flow as function of radius in the outer core. Without needing to replicate the demonstration, let us just write the resulting equation for the convective heat flow:

$$
\begin{aligned}
Q_{\text {conv }}(r) & \equiv \int_{A(r)}\left(\mu^{\prime} \overline{\rho_{a} \xi_{c} \boldsymbol{v}}+T_{a} \overline{\rho_{a} s_{c} \boldsymbol{v}}\right) \cdot \mathrm{d} \boldsymbol{A} \\
& =-\int_{V(r)} \rho_{a}\left(C_{p} \frac{\partial T_{a}}{\partial t}+\mu^{\prime} \frac{\partial \xi}{\partial t}\right) \mathrm{d} V+T_{L}\left(r_{I C}\right) \Delta S \rho_{a}\left(r_{I C}\right) 4 \pi r_{I C}^{2} \frac{\mathrm{d} r_{I C}}{\mathrm{~d} t}-\int_{A(r)} \boldsymbol{q _ { s }} \cdot \mathrm{d} \boldsymbol{A},
\end{aligned}
$$

with $V(r)$ the volume inside the sphere of radius $r$ in the core, $A(r)$ its outward oriented surface. This equation states that the total convective heat flow across $A(r), Q_{\text {conv }}(r)$, which has a contribution from both thermal and compositional convection, is equal to the sum of 

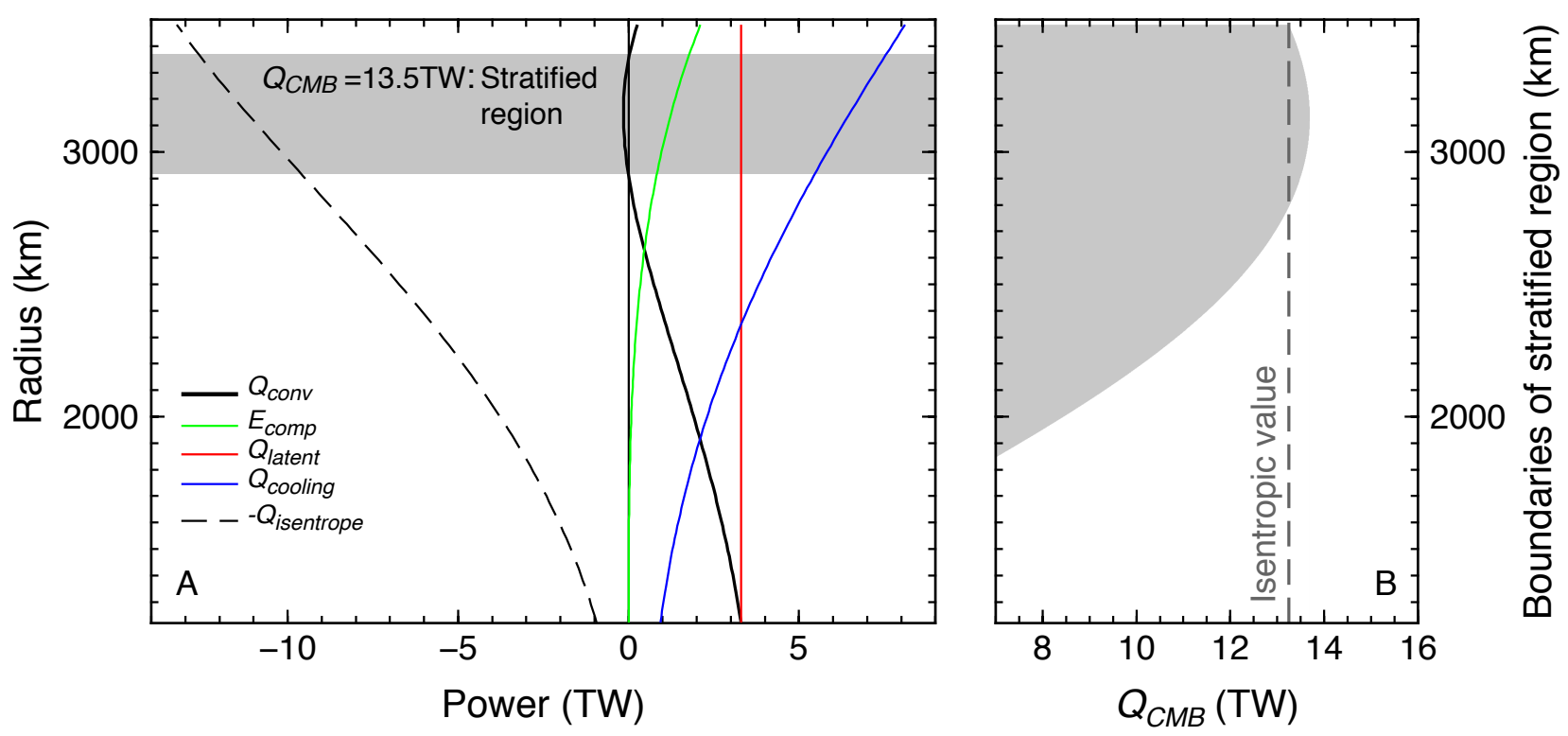

Figure 8: Radial profiles of contributions to the convective heat flow (A) and limits to the region with negative convective heat flow as function of the present time CMB heat flow value (B). The profiles are computed for $Q_{C M B}=13.5 \mathrm{TW}$, that is slightly larger than the isentropic value $\left(Q_{S}=13.25 \mathrm{TW}\right)$.

the energies provided within the sphere $V(r)$ minus what is conducted along the isentrope, $Q_{S}(r)$, the last term on the right-hand-side. The energy "production" terms are, by order of appearance on the right-hand-side, the secular cooling $Q_{C}(r)$, the compositional energy $E_{\chi}(r)$ and the latent heat $Q_{L}$. This equation neglects any contribution from radioactivity for simplicity but its inclusion would be straightforward. Note that each of these terms has exactly the same form as its equivalent for the total core discussed in section 3.1 and indeed for $r$ tending to the radius of the core, $Q_{c o n v}(r)+Q_{S}(r)$ tends toward the CMB heat flow. This means that each term can easily be computed with exactly the same expressions as discussed before and given in the appendix by simply replacing $r_{O C}$ by $r$. It also implies that each source term depends on the inner core radius and is proportional to its growth rate. Therefore, the computation of the convective profile requires the knowledge of the inner core growth rate, that is to say the CMB heat flow.

An example of such a calculation was performed by Gomi et al. (2013) and another one is shown on figure 8A for the present higher order model. The value of the CMB heat flow chosen here, $13.5 \mathrm{TW}$, is slightly above the isentropic value (13.25 TW) and shows the interesting behavior that was first identified by Gomi et al. (2013): In order to maintain the isentropic temperature profile in the whole core, the convective heat flow has to be negative in a region between radii $2900 \mathrm{~km}$ and $3350 \mathrm{~km}$. The reason for this comes from the increase of the thermal conductivity with depth, which makes the density of heat flux along the isentrope present a maximum at intermediate depth (fig. 2) and the surface integrated version decrease less rapidly with depth than it would for a constant conductivity (Gomi et al., 2013).

Is it possible for convection to transport heat downward as is required in this case to maintain the isentropic temperature gradient everywhere? From a thermodynamical point of view, the answer is yes as long as the dissipation stays positive, which is the case of the calculation shown on figure 8A. Dynamically, this can happen because convective motions driven both from cooling at the CMB and release of thermal and compositional buoyancy at the ICB can penetrate in a stably stratified environment. This phenomenon, termed penetrative convection, is well known in the astrophysical context where motions from the stellar convective interior 
can penetrate hundreds of kilometers within the stably stratified radiative layers (eg. Zahn, 1991; Brummell et al., 2002). Using parameters from the core, however, the type of approach developed for stellar interiors leads to estimates of depth of penetration of a few kilometers at most (Gomi et al., 2013), mainly because inertia is much smaller in the core than in the solar interior. Fully dynamical calculations in the case of the core are nevertheless needed to assess the outcome of the potential stratification predicted by energy balance arguments.

The case presented on figure $8 \mathrm{~A}$ shows that a CMB heat flow equal to the isentropic value is not sufficient to ensure a positive convective heat flow in the whole core. If penetration from the surrounding layers is not efficient in the core, a stratified layer would develop at some intermediate depth. Figure 8B shows as shaded area the region that would be stably stratified in that case, as function of the CMB heat flow. In particular, this shows that if the CMB heat flow is exactly equal to the isentropic value, a stably stratified layer about $700 \mathrm{~km}$ thick would exist just below the CMB. A similar behavior was obtained by Gomi et al. (2013) but the thickness of the layer was even larger. On the other hand, the isentropic heat flow was smaller. In any case, this situation is markedly different from what was proposed by early studies of the consequences of a sub-isentropic CMB heat flow (Gubbins et al., 1982; Labrosse et al., 1997; Lister and Buffett, 1998). These studies assumed a constant thermal conductivity so that the maximum isentropic heat flux density was obtained at the CMB in which case the stratified layer thickness shrinks to zero for an isentropic CMB heat flow.
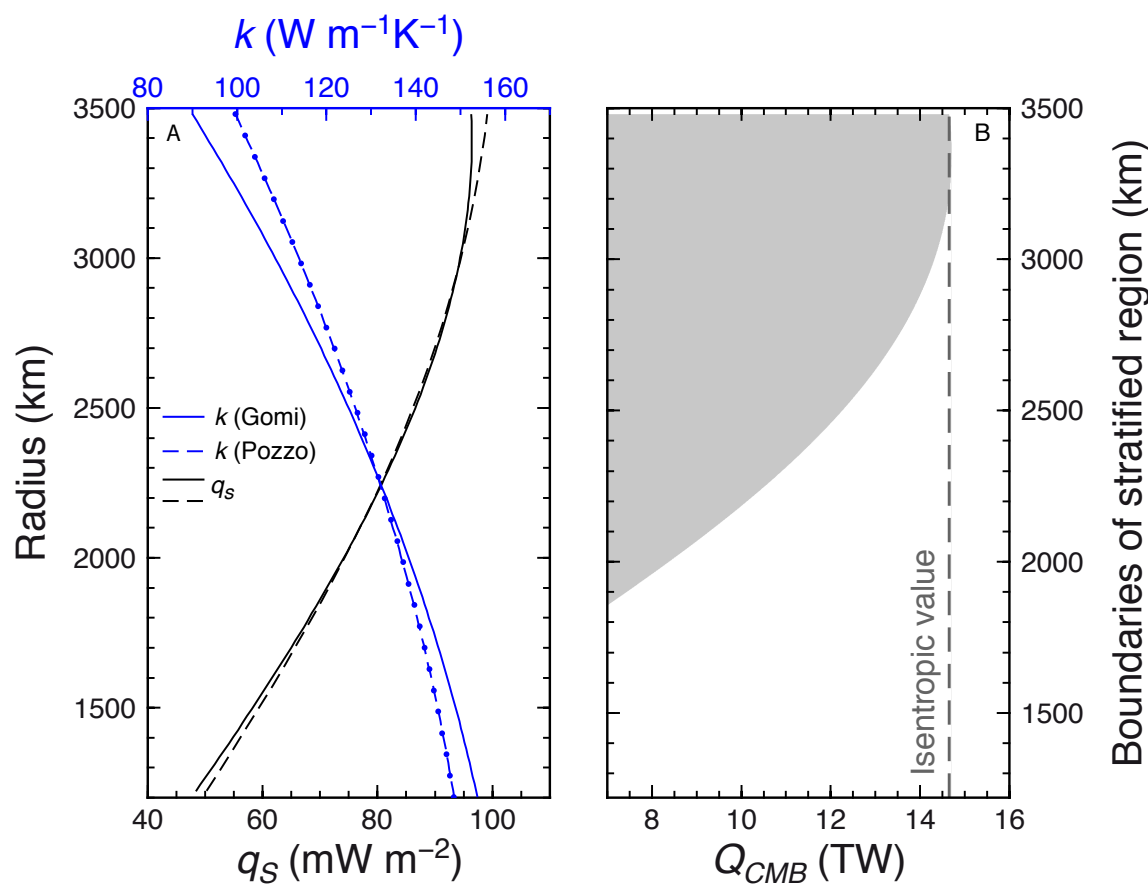

Figure 9: (A) Comparison of profiles of thermal conductivity and isentropic heat flux between the present work and that of Pozzo et al. (2012) and Gubbins et al. (2015). The solid blue line is the same conductivity as that presented on figure 2, for reference, the blue dots are the conductivity values from Pozzo et al. (2012) and the dashed blue line is the fit of these values according to equation (20). The dashed black line is the isentropic heat flux density of Pozzo et al. (2012) while the solid black line is obtained with the temperature profile used in the present study and the conductivity of Pozzo et al. (2012). (B) Radial boundaries of the predicted stratified layer as a function of the CMB heat flow obtained using the conductivity profile of Pozzo et al. (2012).

Note that even though the results depicted on figure 8 are qualitatively the same as that of Gomi et al. (2013), they differ quantitatively. The reason for this lies in the different profiles of density (fig. 1) and therefore temperature. Pozzo et al. (2012) also predicted the possibility 
of a stratified layer at the top of the core, based on a different approach, and did not obtain the behavior just discussed: in their calculation, an isentropic CMB heat flow is sufficient to maintain convection in the whole core. In order to investigate the origin of these differences, I used their conductivity values (dots in figure 9-A) which can be nicely fitted by equation (20) with $k_{0}=99.58 \mathrm{Wm}^{-1} \mathrm{~K}^{-1}$ and $A_{k}=0.35955$ (blue dashed line in figure 9-A). Using the same temperature profile as for the rest of this paper, the profile of isentropic heat flux can be computed (black solid line in figure 9-A) and compared to that of Pozzo et al. (2012) (black dashed line in figure 9-A). The small difference between these two profiles comes from the slightly different density and therefore isentropic temperature profiles: Pozzo et al. (2012) use directly the density polynomial from PREM, instead of the fourth order polynomial used here (eq. 5). Compared to the equivalent isentropic heat flux plotted on figure 2, both presented on figure 9 reach their maximum value at the CMB. The difference is quantitatively small but when the boundaries of the stratified layer are computed in the same way as on figure 8 , the result is qualitatively different: the stratified layer vanishes when the CMB heat flow is super-isentropic.

Comparing the two conductivity profiles on figure 9, it seems clear that their difference is not large and likely within their respective uncertainties. It is therefore difficult to claim that a stratified layer can exist within the core even if the CMB heat flow is slightly super-isentropic. But it is as difficult to claim the opposite and it seems that the details of the conductivity profile need to be worked out before a definite conclusion can be reached on that specific point.

The existence of a stratified layer at the top of the core has been proposed from seismology (eg. Garnero et al., 1993; Tanaka and Hamaguchi, 1993; Tanaka, 2007; Helffrich and Kaneshima, 2010) but its thickness is less than $300 \mathrm{~km}$. In addition, the maximum thickness of a stratified layer at the top of the core is constrained to be less than about 100km from geomagnetic studies (Whaler, 1980; Gubbins, 2007). This means that a sub-isentropic heat flow at the CMB is excluded for the present time unless convection can penetrate about $600 \mathrm{~km}$, which seems rather unlikely, or the gradient of thermal conductivity with depth is lower or equal to that of Pozzo et al. (2012).

Before the existence of the inner core, a similar approach allows us to compute the convective heat flow necessary to maintain an isentropic temperature profile in the whole core (Gomi et al., 2013). The calculation is simpler than in the case discussed above: the heat flow at the CMB is equal to the sum of convective and conductive heat flow crossing $A(r)$ plus the secular cooling of the shell between the radius $r$ and the CMB. The secular cooling can be computed from the global energy balance, assuming the whole core to be isentropic. The result of this calculation is shown on figure 10A. The calculation here is done for a CMB heat flow equal to $16.1 \mathrm{TW}$, while the isentropic value is $14 \mathrm{TW}$. This allows thermal convection in the upper part of the core but maintaining an isentropic profile deeper than the radius $2650 \mathrm{~km}$ requires a downward convective flux. Figure 10B shows the evolution of the upper limit of the stratified core as function of the CMB heat flow. This radius is found to reach 0 for $Q_{C M B}=17.5 T W$ and for a lower value, a large region at the center of the core tends to be stratified. Obviously, for a sub-isentropic CMB heat flow, the whole core is stratified since no other source of buoyancy is available. This means that the isentropic value is a strict minimum for the CMB heat flow before the existence of the inner core which, according to the previous section, is most of the geomagnetic history.

\section{Thermal evolution models}

The energy balance equation presented in section 3 allows one to compute the thermal evolution of the Earth core provided the CMB heat flow is known at all times, which is unfor- 

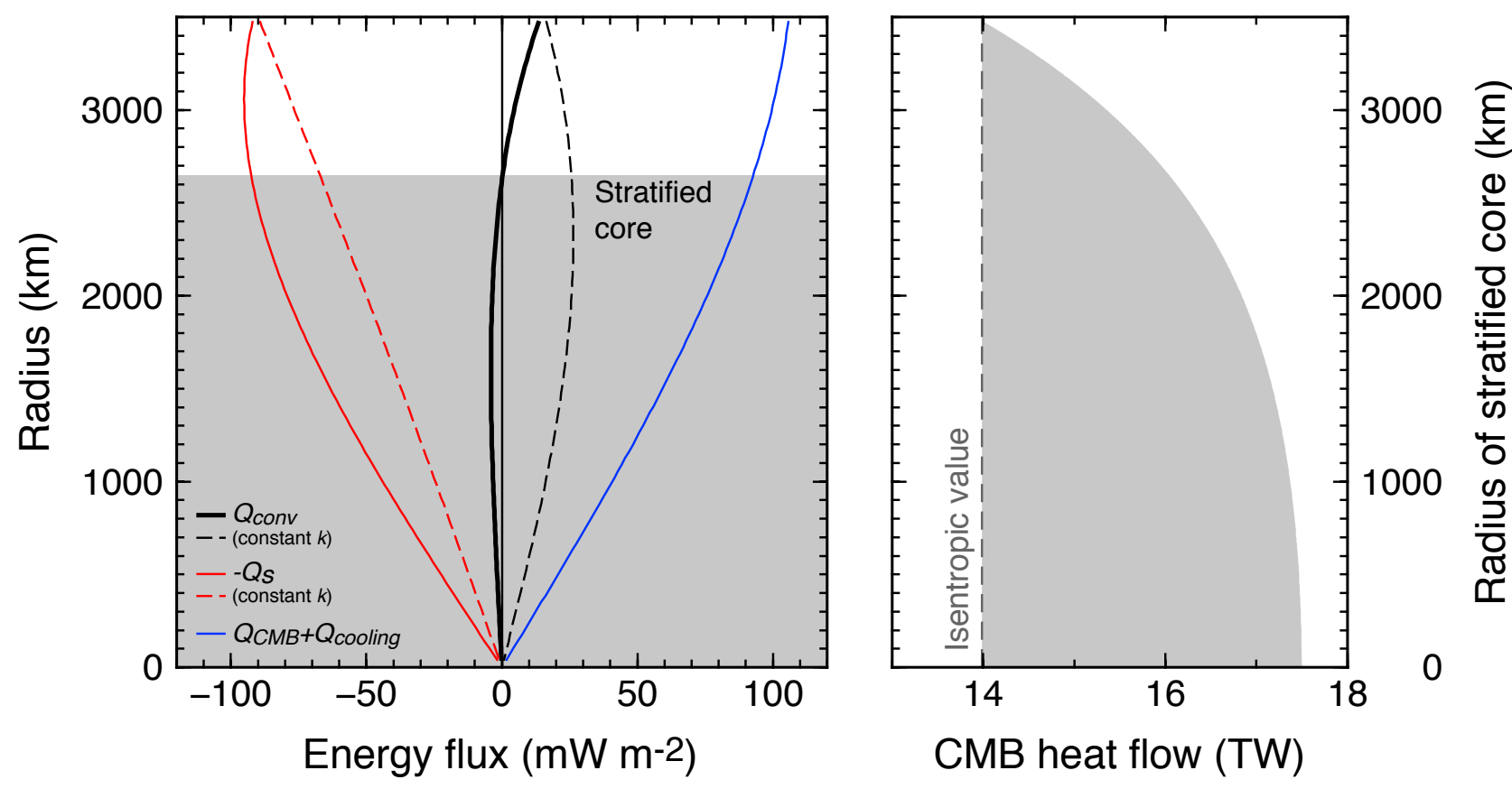

Figure 10: Radial profiles of contributions to the convective heat flux density (A) and limit to the region with negative convective heat flow as function of the CMB value (B) for the period just preceding the onset of inner core crystallization. The profiles are computed for $Q_{C M B}=16.1 \mathrm{TW}$ for an isentropic value $Q_{S}=14 T W$. The dashed lines are obtained for constant value of the thermal conductivity, equal to the CMB value, and show no stratification.

tunately not the case. Even the present value is poorly known. Ideally, it would be desirable to couple a model such as presented here to one for heat transfer across the mantle, either parameterized (eg Stevenson et al., 1983; Mollett, 1984; Grigné and Labrosse, 2001; Nimmo et al., 2004) or fully dynamical (eg. Nakagawa and Tackley, 2010). However, despite important progresses in the last decades, the theory of mantle convection is still far from complete (eg Tackley, 2000; Ricard, 2007; Bercovici and Ricard, 2014) and its efficiency in heat transfer is highly controversial (eg Jaupart et al., 2007). In the present paper I therefore prefer to consider several scenarios for the evolution of the CMB heat flow with time and draw implications from the resulting models. In particular, this allows us to put constraints on the CMB flow from the point of view of the evolution of the core.

In section 3.3, I showed how the energy and entropy balances can be used to infer the CMB heat flow necessary to maintain a given dissipation. The same approach could be used to compute the thermal evolution of the core if the dissipation were known as a function of time. However, the present dissipation is quite uncertain and its time evolution is totally unconstrained from observations. On the other hand, the thermodynamics discussed in the previous sections allows us, within the framework of a fully isentropic core, to impose minimum constraints for the thermal evolution: the total dissipation must be positive, which gives a CMB heat flow larger than 6.9TW at the present time and $16 \mathrm{TW}$ just before the onset of the inner core.

Note however that the minimum value of the CMB heat flow that ensures a positive present dissipation, about 6.9TW (figure 6), is largely sub-isentropic and requires the convective heat flow to be negative in the $1640 \mathrm{~km}$ top of the core. From a thermodynamical point of view, this means that a flow that would operate with negligible dissipation, either viscous or Ohmic, could maintain convection in the whole core, driven from the bottom part and penetrating in the upper part of the core. This is possible thanks to the high efficiency in the conversion of 
compositional energy in dissipation, although rather unlikely. On the other hand, in the case without inner core, the minimum CMB heat flow necessary to maintain a positive dissipation is about $16 \mathrm{TW}$ (figure 4) and is therefore greater that the isentropic value, 14TW. In that case, the super-isentropic top region is required to drive flow in the sub-isentropic inner region. But as discussed in the previous section, the present core is unlikely to operate with a thick stably stratified layer at its top. In addition, having the CMB heat flow drop once the inner core starts crystallizing is unlikely since it is controlled by mantle convection that is not affected by the presence or absence of an inner core. For this reason, the CMB heat flow is assumed here to keep a constant ratio to the isentropic value at all times and only the ratio is used as an input parameter in the thermal evolution models.
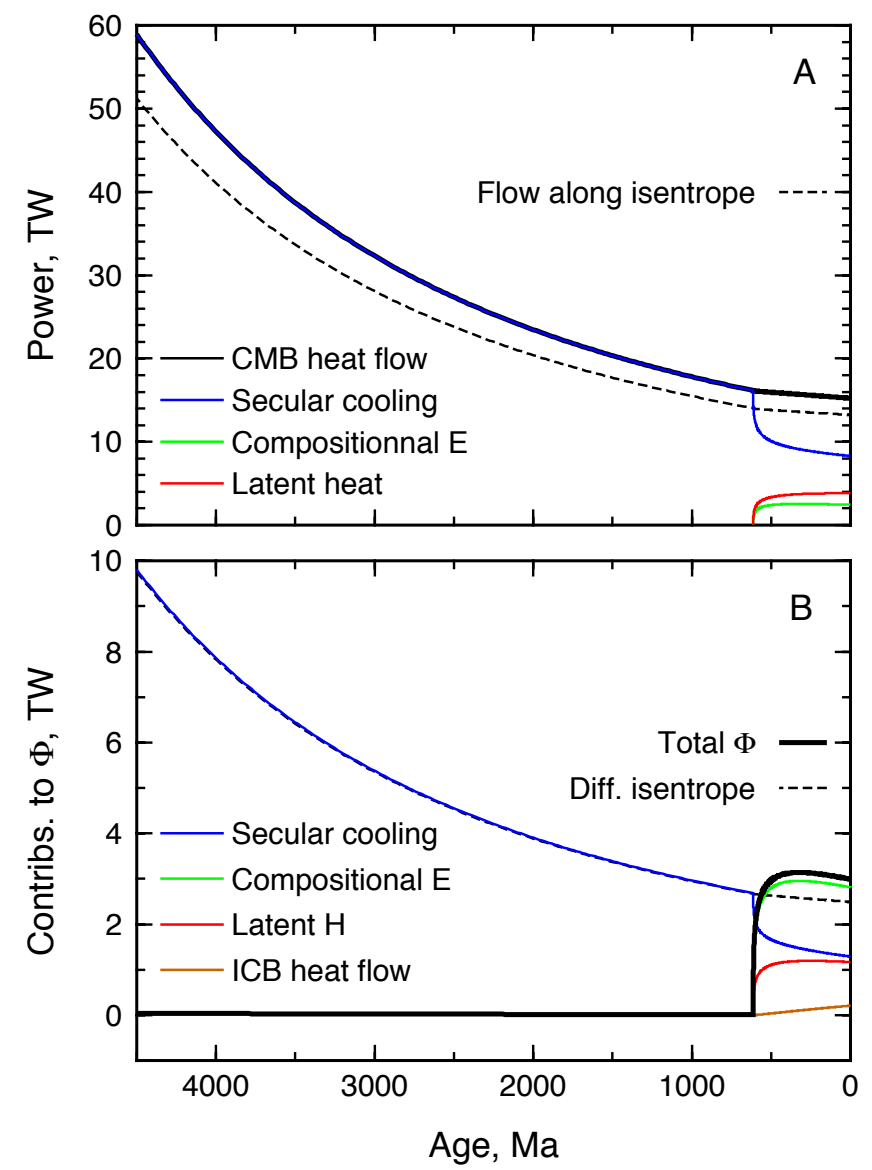

Figure 11: Evolution of the contributions to the the CMB heat flow (A) and total dissipation (B) as function of time for a CMB heat flow that is always 1.15 times the isentropic value.

We have seen (fig. 4) that the minimum CMB heat flow to maintain a positive dissipation just before the onset of the inner core is 1.14 times larger than the isentropic value and figure 11 shows the evolution with time of the contributions to both the total dissipation and the energy budget for a CMB heat flow that is always 1.15 times the isentropic value. The calculation is performed in several steps. Prescribing the value of the CMB heat flow at present and just before the onset of the inner core crystallization and assuming it varies linearly with time between these to periods allows us to compute analytically the age of the inner core. The calculation is approximate since the inner core is assumed to be isentropic but the approximation is very good. Before the existence of the inner core, prescribing a CMB heat flow which is a constant times the isentropic value allows us to solve the evolution analytically. Then, the thermal evolution for the period since the onset of the inner core is solved numerically, in order to obtain a proper 
solution for conduction in the inner core (Labrosse, 2014), which provides the value of the ICB heat flow which, as expected, is small (fig. 11).

Figure 11 shows that indeed this thermal evolution produces a dissipation that is barely positive for the whole period before the onset of inner core crystallization. At that moment, 614 Myr BP, the onset of different types of energy sources to drive convection, and in particular compositional energy, makes the dissipation increase sharply to reach a value around $3 \mathrm{TW}$. This behavior was already discussed (Labrosse, 2003; Labrosse and Macouin, 2003; Aubert et al., 2009) and shows that addressing the question of the minimum value of the dissipation at the present time is not sufficient: because the inner core is so young, the present CMB heat flow is in fact constrained by the minimum requirements before its existence and the present dynamo is likely to operate at a much larger dissipation than the minimum required by thermodynamics. Of course, if it is acceptable despite the potentially very thick stably stratified layer that could result, it is possible that the CMB heat flow became sub-isentropic just after the onset of inner core crystallization. However, the $\mathrm{CMB}$ heat flow results from mantle convection with little retroaction from the dynamics of the core, except insofar as it maintains a nearly uniform temperature at the CMB. Therefore, the variation of the CMB heat flow cannot be affected by the onset of inner core crystallization. This scenario can nevertheless be considered (Aubert et al., 2009) but makes little difference: the age of the inner core is slightly increased and the sharp increase of the total dissipation remains.

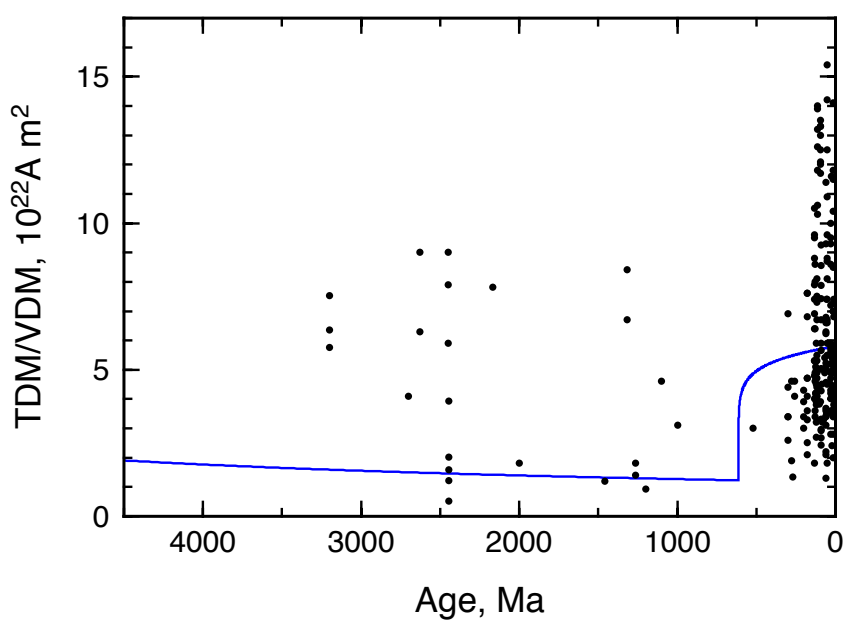

Figure 12: Evolution of the predicted dipole moment for the thermal evolution presented on figure 11 (solid blue line) and of the measured virtual dipole moments (dots, same selection as by Aubert et al., 2009). Note that the expected increase of the dipole field is small compared to the observed fluctuations and would be hardly detectable. See text for a discussion.

Using the same scaling laws as that proposed by Aubert et al. (2009), the evolution of the dissipation shown on figure 11 can be converted into a prediction for the evolution of the magnetic dipole moment and compared to observations from paleomagnetism (fig. 12). The same database as that used by Aubert et al. (2009) is presented here. Because of the $1 / 3$ exponent in the expression of the dipole moment as function of dissipation (Christensen and Aubert, 2006; Aubert et al., 2009), the sharp jump in the dissipation, which is maximized by considering a minimal scenario for the period before the inner core, only implies a factor $\sim 6$ increase in the dipole moment. Because of the large variations (data scatter and intrinsic fluctuations) in the paleointensity measurements, the predicted evolution goes through the measurements. The comparison is interesting however because it shows that the large fluctuations in the dipole field can happen with little variation of the dissipation. The Ohmic dissipation is proportional to the 
square of the electric density, $\phi_{\sigma}=\boldsymbol{J}^{2} / \sigma$, with $\sigma$ the electric conductivity, and $\boldsymbol{J}=\boldsymbol{\nabla} \times \boldsymbol{B} / \mu_{0}$. Therefore, considering a magnetic field varying on a lengthscale $l, \boldsymbol{B}(l)$, its contribution to the total dissipation is of order $(B / l)^{2} / \mu_{0}^{2} \sigma$. This means that the contribution of the dipole to the total dissipation is small (see Roberts et al., 2003, for a more complete discussion) and it can fluctuate on short time scales without much constraints from the thermodynamical balances.

Another message that can be obtained from figure 12 is that it appears difficult to accept any significant period of time without magnetic field since $3.5 \mathrm{Gyr}$. Based on the sole data presented on the figure, which where selected by Aubert et al. (2009) on the basis of their accuracy for paleointensity measurements, we might fit a couple of periods, a few Myr long, where a null field could have prevailed but in fact these periods shrink when considering the full database. The importance of this observation comes from the expected fluctuations of the CMB heat flow as predicted by mantle convection models. For example, in the study of Nakagawa and Tackley (2010), the calculations that yield a final inner core size closest to the actual one are obtained for the lowest CMB heat flow and the fluctuations resulting from the intrinsic time-dependence of mantle convection make the magnetic field experience long periods of extinction. Independently of any specific mantle convection model, important $(\sim 30 \%)$ fluctuations of the CMB heat flow are expected, if the bottom boundary layer of the mantle is influenced by the arrival of subducted plates (Labrosse, 2002) whose dynamics is highly variable (eg. Labrosse and Jaupart, 2007; Loyd et al., 2007; Becker et al., 2009; Coltice et al., 2012). And the fluctuations that are discussed here concern the full CMB heat flow, not the much smaller fraction that is above the core isentropic value. This means that if indeed the geodynamo has been operating continuously for the last $3.5 \mathrm{Gyr}$, we must account for the fluctuation of the $\mathrm{CMB}$ heat flow and consider a value about $30 \%$ larger than the minimum considered above.

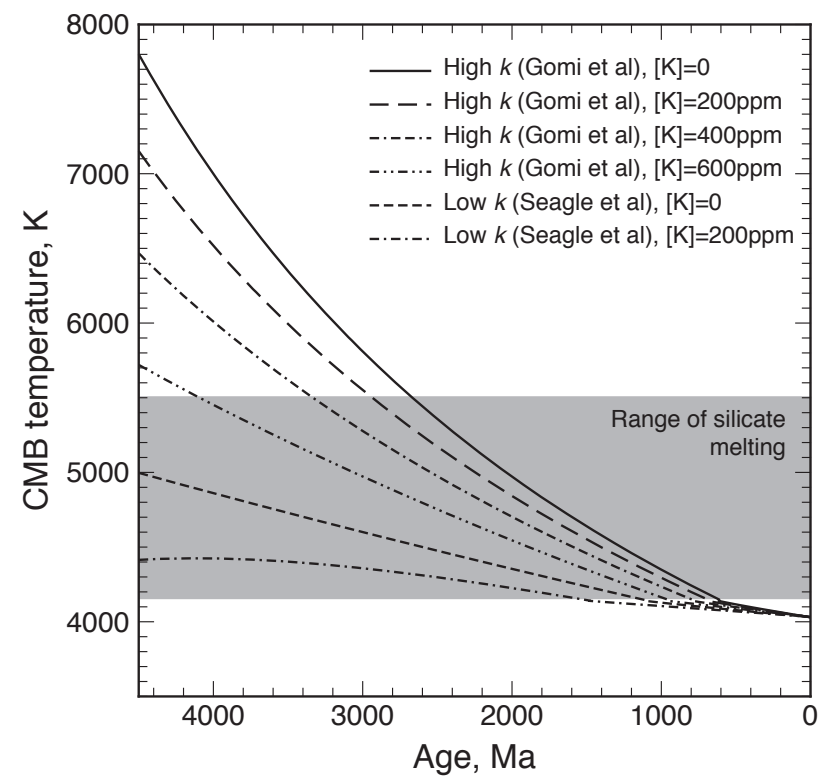

Figure 13: Evolution of the CMB temperature with time for the model presented on figure 11 (solid line) as well as cases with varying amounts of potassium in the core and for the conductivity value proposed by Seagle et al. (2013), as labeled. The shaded area corresponds to the approximate range between the solidus and the liquidus for a "chondritic mantle" (Andrault et al., 2011) or a peridotite (Fiquet et al., 2010).

Finally, the thermal evolution model presented above provides the evolution of the CMB temperature with time (fig. 13). The apparition of the inner core is marked by a decrease of the cooling rate since part of the CMB heat flow is then used to extract latent and compositional 
energies. Note that the effect of radioactivity on the age of the inner core is limited, even with the very large values concentrations of potassium considered here. The initial temperature from the calculation corresponding to figure 11 (solid line) is about $7800 \mathrm{~K}$ and is well above the liquidus of peridotite and a "chondritic" mantle, as determined by Fiquet et al. (2010) and Andrault et al. (2011), respectively. This is not a surprise that mantle melting would be predicted from calculations of the evolution of the core since current presence of partial melt at the bottom of the mantle has been invoked to explain the occurrence of ultra low velocity zones (ULVZ) (eg Williams and Garnero, 1996; Rost et al., 2005). The present CMB temperature is already very close to the solidus, or even higher for basalt compositions (Andrault et al., 2014), and even milder core evolution calculations would predict significant melting of the lower mantle in the past. This is the reason behind the basal magma ocean model (Labrosse et al., 2007) which argues that ULVZs are the remnant of a once much thicker magma ocean. In view of that model, comparing the evolution of the CMB temperature to the solidus and liquidus of bulk mantle compositions is not enough since the actual composition of the magma is likely to evolve with time (eg. Thomas et al., 2012).

Inclusion of radiogenic heating in the picture decreases the initial temperature. As shown on figure 13 , the CMB temperature at $4.5 \mathrm{Gyr}$ is equal to $7150 \mathrm{~K}, 6500$ and $5720 \mathrm{~K}$ for a potassium content of $200 \mathrm{ppm}, 400 \mathrm{ppm}$ and $600 \mathrm{ppm}$, respectively. It has long been considered that the core could contain some potassium but the maximum amount is limited by the its partitioning behavior between metal and silicate during core formation. Rama Murthy et al. (2003) find that the maximum possible concentration of potassium in the core is $130 \mathrm{ppm}$, obtained if sulfur is the only light alloying element responsible for the core density deficit with respect to pure Fe. Nomura and Hirose (2014) studied more generally the partitioning behavior of different light elements and of potassium and find that a maximum of $20 \mathrm{ppm}$ is allowed in the core. Additionally, the abundance of potassium in the silicate Earth relative to chondrite is in par with other moderately volatile elements, suggesting that its budget does not allow a significant amount to be sequestered in the core (McDonough, 2003). The range of concentration considered here is therefore much broader than considered likely based on geochemical and mineralogical arguments. Nevertheless, even 600ppm of $\mathrm{K}$ is not sufficient to avoid melting a large part of the lower mantle in the early Earth (fig. 13). With the high values of thermal conductivity recently published, a much larger radioactive heating rate is necessary if one wants to balance the heat flow that is necessary to maintain the dynamo.

Figure (13) also shows the results of calculations using a lower thermal conductivity, $k=$ $50 \mathrm{Wm}^{-1} \mathrm{~K}^{-1}$, in the middle of the range proposed by Seagle et al. (2013), without radioactivity or with 200ppm of potassium. This shows that the value of the thermal conductivity has a much more important effect on the projected initial temperature of the core than the amount of radioactivity in bounds limited by geochemistry. Nevertheless, even the much lower value of thermal conductivity is not sufficient to avoid melting of the lower mantle in the deep past because the present CMB temperature is close to the solidus of mantle silicates and cooling of the core is necessary to maintain a convective geodynamo.

\section{Discussion and conclusions}

The theory presented above allows us to compute the requirements for running the geodynamo for a fully isentropic and compositionally well mixed outer core. This assumption comes in the calculation of the average structure and allows computation of the different terms in the energy and entropy balances. With these assumptions, we can get the minimum value of the CMB heat flow as function of time and compute the thermal evolution with that minimum value. Figure 11A shows the evolution of the minimum CMB heat flow, which is much larger 
than usually assumed (eg. Lay et al., 2008). This large heat flow implies, by conservation of energy, a high initial core temperature (fig. 13). The large CMB heat flow results from the large value of the thermal conductivity obtained in recent studies (de Koker et al., 2012; Pozzo et al., 2012; Gomi et al., 2013; Pozzo et al., 2014). The present results can be considered minimum since the conductivity value used is the minimum proposed by Gomi et al. (2013) and corresponds to the case where $\mathrm{Si}$ is the sole light element in the core. In the more likely case that a mixture of $\mathrm{Si}, \mathrm{S}$ and $\mathrm{O}$ is present, we expect a somewhat larger value. Note that Seagle et al. (2013) did similar experiments as Gomi et al. (2013) and obtained similar values for the electrical resistivity at low temperature. However, they proposed a smaller value for the thermal conductivity after extrapolation to high temperature. The reason for this discrepancy comes from their not considering the effect of saturation of the resistivity at high temperature, an effect that is well documented in the metallurgy literature (see Gomi et al., 2013, for a discussion and references) and recently confirmed for iron at high pressure (Ohta et al., 2014).

The initial temperature predicted by the current model and shown on figure 13 is quite large and, if compared to the liquidus of potential bulk mantle compositions and extrapolated upward isentropically, might predict that the whole mantle has been fully molten for a long period of its early history. That would be problematic since the cooling of the magma ocean is expected to happen on a few Myr timescale (eg. Abe, 1997; Sleep, 2000). But evidence for the existence of a geomagnetic field dates to 3.5 Gyr (Usui et al., 2009; Tarduno et al., 2010) and it may indeed not have existed before. In fact, it has been argued on the base of geochemical analysis of the lunar soil that the Earth has lacked a magnetic field until 3.9 Ga (Ozima et al., 2005). Also, the basal magma ocean model tends to predict that the onset of dynamo activity could be delayed (Labrosse et al., 2007). In that case, the early core could have been stably stratified which would remove the need for the long initial cooling rate. Also, only the CMB temperature is constrained by the present model and for the thermal structure of the implied magma ocean to be computed, the Grüneisen coefficient is required. Experimental (Mosenfelder et al., 2007) and ab initio calculations (Stixrude et al., 2009) studies have shown the Grüneisen parameter of some silicate liquids increase strongly with pressure and could lead to a steep isentropic temperature gradient. Additionally, the bulk mantle composition that is considered today is based on observations made today, after 4.5 Gyr of cooling, fractional crystallization and remixing of the freezing products. This means that even at the highest temperature obtained at $3.5 \mathrm{Gyr}$, the isentropic temperature profile in the magma ocean could cross the liquidus of the most refractory minerals which then have been already solidifying and possibly floating up.

Another issue concerns the assumption of an isentropic temperature gradient in the whole liquid core. The situations in which a negative (ie. downward) convective heat flow is required arrises in order to maintain an isentropic temperature profile. The possibility of a sub-isentropic CMB heat flow has been mentioned for several decades (Loper, 1978; Gubbins et al., 1979; Stacey and Loper, 1984; Buffett et al., 1996; Gubbins et al., 2004, 1982; Labrosse et al., 1997; Lister and Buffett, 1998) even though the thermal conductivity was assumed much smaller than now, because the CMB heat flow was also assumed much smaller. Most models assumed, as done in the preceding sections, that compositional convection was nevertheless able to maintain the isentropic gradient by transporting heat downward, which was termed the "refrigerator effect". A few studies departed from this assumption and considered alternatively that sub-isentropic regions could become thermally stratified (Gubbins et al., 1982; Labrosse et al., 1997; Lister and Buffett, 1998; Buffett, 2007). In these studies, the thermal conductivity was considered uniform and the isentropic heat flux was maximum at the surface. For this reason, when the CMB heat flow dropped below the isentropic value, a conduction layer was developing at the 
top and, starting from a zero thickness, would grow with time for a decreasing CMB heat flow. The temperature profile in the stratified layer evolved according to the conduction equation which made the temperature gradient smaller than the isentropic one, therefore reducing the contribution of conduction to the entropy budget, leaving a larger fraction for dynamo action. Similar models could be developed for the situation considered here, which could help to lessen the constraints on the CMB heat flow. However, the difference would not be large enough to drastically change the outcome. Inspection of figure $4 \mathrm{~A}$ shows that the difference between the situation with a zero dissipation, where convection from the top unstable layer is assumed to penetrate deep in the stratified core, and the isentropic heat flow, which would drive convection in an infinitely thin layer at the top of the core, is only 2 TW. This means that even if we considered, as an extreme end-member, that the core has a uniform temperature below the convecting region, therefore removing entirely the toll of diffusion along the isentrope, a CMB heat flow larger than the isentropic value would still be necessary to drive the dynamo and this heat flow is only $2 \mathrm{TW}$ less than what considered before, 17TW. Of course, in that case the dissipation would be larger but that does not change considerably the amount of cooling experienced by the core necessary to explain its thermal history.

Gubbins et al. (2015, this issue) suggest that the geodynamo could operate with an average sub-isentropic CMB heat flow provided that some locations have a super-isentropic heat flux. Clearly, large lateral variations of heat flux are expected at the CMB from mantle convection, as they are observed at the Earth surface (Jaupart et al., 2007), and for any reasonable value of the average CMB heat flux, we should expect regions of the CMB where the heat flux is super-isentropic and regions where it is sub-isentropic. This must influence core dynamics (eg. Aubert et al., 2007) and deserves discussion here. The main problem is for the period before the existence of the inner core which also largely controls the initial CMB temperature. A subisentropic average CMB heat flux would imply a thermal stratification in the whole core since the total CMB entropy flux, $\int_{A\left(r_{O C}\right)}(\boldsymbol{q} / T) \cdot \mathrm{d} \boldsymbol{A}$, would not allow the production of entropy by conduction along the isentropic temperature gradient to be maintained. Nevertheless, lateral variations of the CMB heat flux would drive a thermal wind at the top of the core and the question is whether this could maintain a dynamo. From a thermodynamics perspective, this is possible but not very efficient. The efficiency equation (40) is still valid but its implications are potentially different. Having a sub-isentropic temperature gradient reduces the toll from conduction by making $S_{k}$ lower on the right hand side. On the other hand, it also reduces the efficiency factor of the cooling term by reducing the temperature difference between the bulk of the core where cooling happens and the CMB. In fact, conductive cooling which should dominate in that situation concentrates at the top of the core and the relevant temperatures entering the efficiency factor, $T_{C}, T_{\Phi}$ and $T_{C M B}$ are all close to each other making the Carnotlike efficiency ratio small. Whether it is sufficient to maintain a dynamo able to explain the observations from paleomagnetism can only be studied using complete geodynamo models.

The discussion above focused on the minimum CMB heat flow, and therefore temperature evolution, in order to maintain the thermodynamic possibility of running a dynamo. That raises several questions that all derive from one: what is in fact the minimum CMB heat flow necessary to explain the paleomagnetic observations? Figure 12 shows that, using scaling laws from geodynamo models to predict the evolution of the dipole field with the minimum scenario leads to acceptable intensities. But this requires that the CMB heat flow never drops below the minimum necessary to maintain the dynamo. Considering that the CMB heat flow is controlled by mantle convection and therefore should fluctuate by about $30 \%$ around its long term evolution on a several 100s Myr time-scale (eg. Nakagawa and Tackley, 2010), the demand on the CMB heat flow should be raised by about 30\%. Unless of course periods of dynamo 
extinction can be fitted in the observations. In other words, it appears necessary not only to constrain the timing of the dynamo onset (Tarduno et al., 2010), but also to investigate whether its operation has been uninterrupted since. These are clearly difficult questions to answer since absence of evidence does not mean evidence of absence and the scarcity of the paleomagnetic record for old ages is largely due to alteration of samples.

What are the options if the demand on the CMB heat flow and the initial temperature is deemed too high with the current model and parameters? We can first question some of the parameters. For example, the Grüneisen parameter used in the present study comes from the ab initio calculations of Vočadlo et al. (2003) who found it to be almost constant along the core isentrope. Ichikawa et al. (2014) found it to decrease slightly with depth in the core which would make the contribution of conduction to the entropy equation smaller. This is not expected to change the results by much since, as discussed above, the difference between the CMB heat flow necessary to maintain a null dissipation and the isentropic heat flow is small. The CMB value of the Grüneisen parameter is the most important value to consider since it controls the isentropic heat flow. The value of Ichikawa et al. (2014), 1.45, is only $3 \%$ smaller than that used here and this difference is not sufficient to change the results.

The density difference across the ICB is highly uncertain (Hirose et al., 2013) but the value used here is on the high end of the current estimates. Using a smaller value would make the compositional energy smaller and increase the demand on other sources, therefore on the cooling rate of the core. However, this only matters for the period since the onset of the inner core but the main difficulty comes from the much longer period before that.

The main parameter that makes a difference is the thermal conductivity. However, as already discussed, the value considered here is conservative since it is in the low end of the estimates from the several independent recent studies. If one wants to decrease the demand on core cooling of the dynamo, a totally different driving mechanism is required. The theory developed here assumes that the geodynamo results from thermal and/or compositional convection in a well mixed isentropic environment. Moreover, compositional convection is assumed to happen only from release of light elements upon inner core crystallization and chemical interaction between the core and the mantle have been neglected. Other scenarios have been proposed in the recent years that need to be discussed.

The fact that compositional convection is assumed to only happen because of inner core growth is the major limiting aspect of the standard model. If light elements where able to escape the core and enter the mantle, it would leave the upper part of the core denser than the bulk and would drive compositional convection from above. Buffett et al. (2000) proposed that such depletion could happen as a sedimenting process. That model was mostly proposed to explain ultra low velocity zones and to offer a mechanism to create a highly conducting layer at the bottom of the mantle, therefore allowing electromagnetic coupling of the core and mantle. Assuming that the light elements that sediment at the top of the core are the ones that get released from the inner core, the authors estimated that the compositional energy from inner core growth would be doubled. In this scenario, the additional energy source exists only for the period with a growing inner core. Since the compositional energy is the largest contributor to the dissipation, a slower inner core crystallization could run the same dynamo, therefore requiring a smaller CMB heat flow. However, if we consider the isentropic heat flow as a minimum in order to avoid a thick stably stratified layer at the top of the core, the energy budget would not be greatly affected by the doubling of the compositional energy (fig. 6) and the main part of the thermal history would still be driven by thermal convection with no significant change.

This idea can however be extended to the early Earth, as proposed by Stevenson (2007). 
If, during core formation, equilibration of metal and silicates occurred at sufficiently high temperature so that a large amount of light elements were incorporated in the core, its subsequent cooling would provoque exsolution of these light elements and their rise to the surface could drive the dynamo. This scenario has not yet been fully explored, notably because most constraints to support it are missing both from high pressure physics and geophysical observations. The main interest of this scenario is that it would offer an alternative energy source for the dynamo in the early Earth. Additionally, if a larger concentration of impurities was present in the core in the early Earth, the thermal conductivity would have been lower, making the requirement to run a dynamo less demanding. The main drawback is that it still requires cooling for exsolution to happen, although maybe less than what is computed with the standard model. Also, high pressure experiments of reaction between iron and perovskite show that a present equilibrium would have much more light elements in the core than can explain the core density (Ozawa et al., 2009; Hirose et al., 2013, and reference therein). This means that, if any compositional interaction is happening across the CMB, it should be that of dissolution of light elements into the core and the not the opposite one. Stevenson (2007) argued for Mg and data from mineral physics are still missing for that, which leaves some room for this alternate scenario to be explored.

Alternatively, the geodynamo could be maintained by other sources of motions like precession or tides (eg. Tilgner, 2005; Cébron et al., 2010; Le Bars et al., 2015). An interesting finding of Cébron et al. (2010) is that the presence of a stably stratified temperature gradient actually promotes tidal instabilities. The interplay between stable and unstable layers in the presence of tides or precession has not been studied so far and in particular the resulting thermal structure is unknown but it has the potential of changing the way we envision the evolution of the core. This should be the subject of future studies.

Another implication of the large thermal conductivity obtained in recent studies concerns other planets. Venus has very similar global characteristics as the Earth but lacks an internally generated magnetic field. Nimmo (2002) argued that this can be explained by the absence of plate tectonics on Venus which makes the CMB heat flow lower. The analysis presented here shows that, indeed, the CMB heat flow required to maintain a dynamo in an Earth-size planet is much larger than previously thought and the absence of subduction on Venus could get it below the threshold, even if an inner core is currently present and slowly growing. Alternatively, in the case with a growing inner core, a dynamo could in fact be operating in the deepest part of the core, driven by compositional energy, but a thick stably stratified upper core could be masking it from detection at the surface.

\section{Acknowledgements}

This work was completed while I was still supported by the Institut Universitaire de France. I would also like to express my gratitude to John Hernlund and Kei Hirose for organizing the great Onsen II workshop and inviting me to participate. The need to develop a higher order structure model for the core emerged while discussing the value of the isentropic temperature gradient at the CMB with David Gubbins, Chris Davies and John Hernlund during the workshop. I also want to thank Dave Gubbins for his constructive and review and for providing the data necessary to compute figure 9. A thorough anonymous review also helped to clarify the paper. 


\section{Appendix A. Expressions of the different energy and entropy terms}

\section{Appendix A.1. A useful function}

In many instances, global quantities like mass, energy and entropy involve the integral of the density profile (eq. 5) at some power. It is therefore convenient to introduce the function

$$
\begin{aligned}
\mathrm{f}_{\mathrm{C}}(x, \delta) & \equiv 3 \int\left(1-x^{2}-A_{\rho} x^{4}\right)^{1+\delta} x^{2} \mathrm{~d} x \\
& =x^{3}\left[1-\frac{3}{5}(\delta+1) x^{2}-\frac{3}{14}(\delta+1)\left(2 A_{\rho}-\delta\right) x^{4}+O[x]^{6}\right] .
\end{aligned}
$$

Note that in the case $\delta=0$, equation (A.2) is in fact exact. In other cases, a limited development is used and the error amounts to less than $0.5 \%$, which is lower than the typical uncertainty in many of the physical parameters. The function $\mathrm{f}_{\mathrm{C}}$ is first used to compute the mass of any subshell in the core by direct integration of the density. For example for the whole outer core:

$$
\begin{aligned}
M_{O C} & =4 \pi \rho_{0} \int_{r_{I C}}^{r_{O C}}\left(1-\frac{r^{2}}{L_{\rho}^{2}}-A_{\rho} \frac{r^{4}}{L_{\rho}^{4}}\right) r^{2} \mathrm{~d} r, \\
& =\frac{4 \pi}{3} \rho_{0} L_{\rho}^{3}\left[\mathrm{f}_{\mathrm{C}}\left(\frac{r_{O C}}{L_{\rho}}, 0\right)-\mathrm{f}_{\mathrm{C}}\left(\frac{r_{I C}}{L_{\rho}}, 0\right)\right] .
\end{aligned}
$$

This is used, in particular, in equation (13).

\section{Appendix A.2. Latent heat}

The latent heat term is already given in equation (24) and gives

$$
\mathrm{P}_{\mathrm{L}}\left(r_{I C}\right)=4 \pi r_{I C}^{2} T_{L}\left(r_{I C}\right) \rho_{a}\left(r_{I C}\right) \Delta S
$$

The integrated latent extracted since the onset of inner core crystallisation is obtained simply by integration (eq. 44) to give, keeping terms up to order 2 in $r_{I C} / L_{\rho}$,

$$
\mathcal{L}=\frac{4 \pi}{3} \rho_{0} T_{L 0} \Delta S r_{I C}^{3}\left[1-\frac{3}{5}\left(1+\frac{K_{0}}{T_{L 0}} \frac{\partial T_{L}}{\partial P}\right) \frac{r_{I C p}^{2}}{L_{\rho}^{2}}+\frac{\xi_{O 0}}{2 \mathrm{f}_{\mathrm{C}}\left(\frac{r_{O C}}{L_{\rho}}, 0\right) T_{L 0}} \frac{\partial T_{L}}{\partial \xi} \frac{r_{I C p}^{3}}{L_{\rho}^{3}}\right]
$$

\section{Appendix A.3. Secular cooling}

The secular cooling is obtained from its definition (eq.(22)), the expression of the isentropic temperature profile (eq. 11) with, for $T_{c}$, the liquidus at the ICB (eq. 14) and the expression for the density profile (eq. 5) used also to give the time varying value at the ICB, $\rho_{c}$. After a few simple derivations, one gets

$$
\mathrm{P}_{\mathrm{C}}\left(r_{I C}\right)=-\frac{4 \pi C_{p}}{\rho_{a}^{\gamma}\left(r_{I C}\right)}\left[\frac{d T_{L}}{d r_{I C}}+\frac{2 \gamma \rho_{0} T_{L}\left(r_{I C}\right) r_{I C}}{\rho_{a}\left(r_{I C}\right) L_{\rho}^{2}}\left(1+2 A_{\rho} \frac{r_{I C}^{2}}{L_{\rho}^{2}}\right)\right] \int_{r_{I C}}^{r_{O C}} r^{2} \rho_{a}^{\gamma+1}(r) \mathrm{d} r .
$$

For non-integer values of $\gamma$, the integral in equation (A.7) cannot be obtained analytically but it can obviously be computed numerically or, as done here, by using a polynomial development, 
the difference between the two options amounting to about $2 \%$. The polynomial expression gives, to the same order as before,

$$
\begin{aligned}
\mathrm{P}_{\mathrm{C}}\left(r_{I C}\right)=-\frac{4 \pi}{3} \rho_{0} C_{p} L_{\rho}^{3}\left(1-\frac{r_{I C}^{2}}{L_{\rho}^{2}}-A_{\rho} \frac{r_{I C}^{4}}{L_{\rho}^{4}}\right)^{-\gamma}\left[\frac{d T_{L}}{d r_{I C}}+\frac{2 \gamma T_{L}\left(r_{I C}\right) r_{I C}}{L_{\rho}^{2}} \frac{1+2 A_{\rho} \frac{r_{I C}^{2}}{L_{\rho}^{2}}}{1-\frac{r_{I C}^{2}}{L_{\rho}^{2}}-A_{\rho} \frac{r_{I C}^{4}}{L_{\rho}^{4}}}\right] \\
\times\left[\mathrm{f}_{\mathrm{C}}\left(\frac{r_{O C}}{L_{\rho}}, \gamma\right)-\mathrm{f}_{\mathrm{C}}\left(\frac{r_{I C}}{L_{\rho}}, \gamma\right)\right] .
\end{aligned}
$$

In equation (A.8), $T_{L}\left(r_{I C}\right)$ and $d T_{L} / d r_{I C}$ are obtained from equation (14). For a simplified model assuming the inner core to be isentropic, the integration in equation (A.7) on the whole core, and we get simply

$$
\begin{aligned}
\tilde{\mathrm{P}}_{\mathrm{C}}\left(r_{I C}\right)=-\frac{4 \pi}{3} \rho_{0} C_{p} L_{\rho}^{3}\left(1-\frac{r_{I C}^{2}}{L_{\rho}^{2}}-A_{\rho} \frac{r_{I C}^{4}}{L_{\rho}^{4}}\right)^{-\gamma} & {\left[\frac{d T_{L}}{d r_{I C}}+\frac{2 \gamma T_{L}\left(r_{I C}\right) r_{I C}}{L_{\rho}^{2}} \frac{1+2 A_{\rho} \frac{r_{I C}^{2}}{L_{\rho}^{2}}}{1-\frac{r_{I C}^{2}}{L_{\rho}^{2}}-A_{\rho} \frac{r_{I C}^{4}}{L_{\rho}^{4}}}\right] } \\
& \mathrm{f}_{\mathrm{C}}\left(\frac{r_{O C}}{L_{\rho}}, \gamma\right) .
\end{aligned}
$$

This is the option chosen when computing the age of the inner core.

The computation of $\mathcal{C}$ requires the integration of $\tilde{\mathrm{P}}_{\mathrm{C}}\left(r_{I C}\right)$. This can be done numerically or, as done here, by developing the expression in $r_{I C} / L_{\rho}$. Keeping terms up to second order in $r_{I C} / L_{\rho}$, one gets

$$
\mathcal{C}=\frac{4 \pi}{3} \rho_{0} C_{p} L_{\rho} r_{I C}^{2} \mathrm{f}_{\mathrm{C}}\left(\frac{r_{O C}}{L_{\rho}}, \gamma\right)\left(\frac{\partial T_{L}}{\partial P} K_{0}-\gamma T_{L 0}-\frac{\partial T_{L}}{\partial \xi} \frac{\xi_{O 0}}{\mathrm{f}_{\mathrm{C}}\left(\frac{r_{O C}}{L_{\rho}}, 0\right)} \frac{r_{I C p}}{L_{\rho}}\right)
$$

Comparison of the numerical integration of the full expression (eq. A.9) and the result of equation (A.10) show a difference of less than $3 \%$.

The entropy contribution of the secular cooling is expressed using its definition (eq. 30) and gives

$S_{C}=-\frac{4 \pi \rho_{0} C_{p} L_{\rho}^{3}}{3 T_{L}\left(r_{I C}\right)}\left[\frac{d T_{L}}{d r_{I C}}+\frac{2 \gamma T_{L}\left(r_{I C}\right) r_{I C}}{L_{\rho}^{2}} \frac{1+2 A_{\rho} \frac{r^{2}}{L_{\rho}^{2}}}{1-\frac{r_{I C}^{2}}{L_{\rho}^{2}}-A_{\rho} \frac{r_{I C}^{4}}{L_{\rho}^{4}}}\right]\left[\mathrm{f}_{\mathrm{C}}\left(\frac{r_{O C}}{L_{\rho}}, 0\right)-\mathrm{f}_{\mathrm{C}}\left(\frac{r_{I C}}{L_{\rho}}, 0\right)\right] \frac{\mathrm{d} r_{I C}}{\mathrm{~d} t}$.

Using equation (A.8), the temperature $T_{C}$ at which secular cooling effectively contributes to the entropy balance is

$$
T_{C}=T_{L}\left(r_{I C}\right)\left(1-\frac{r_{I C}^{2}}{L_{\rho}^{2}}-A_{\rho} \frac{r_{I C}^{4}}{L_{\rho}^{4}}\right)^{-\gamma} \frac{\mathrm{f}_{\mathrm{C}}\left(\frac{r_{O C}}{L_{\rho}}, \gamma\right)-\mathrm{f}_{\mathrm{C}}\left(\frac{r_{I C}}{L_{\rho}}, \gamma\right)}{\mathrm{f}_{\mathrm{C}}\left(\frac{r_{O C}}{L_{\rho}}, 0\right)-\mathrm{f}_{\mathrm{C}}\left(\frac{r_{I C}}{L_{\rho}}, 0\right)} .
$$




\section{Appendix A.4. Compositional energy}

The compositional term is obtained by direct integration of its expression (eq.(25)) using the expression for the density (eq.(5)), chemical potential (eq.(18)) and the mass fraction (eq.(13) which gives

$\mathrm{P}_{\chi}\left(r_{I C}\right)=8 \pi^{2} \xi_{O 0} G \rho_{0}^{2} \beta \frac{r_{I C}^{2} L_{\rho}^{2}}{\mathrm{f}_{\mathrm{C}}\left(\frac{r_{O C}}{L_{\rho}}, 0\right)} \int_{\frac{r_{I C}}{L_{\rho}}}^{\frac{r_{O C}}{L_{\rho}}} x^{2}\left(1-x^{2}-A_{\rho} x^{4}\right)\left(x^{2}-\frac{r_{I C}^{2}}{L_{\rho}^{2}}\right)\left[1-\frac{3}{10}\left(x^{2}+\frac{r_{I C}^{2}}{L_{\rho}^{2}}\right)\right] \mathrm{d} x$.

This expression being polynomial can obviously be computed exactly but in the interest of simplification, one can use the leading order development,

$$
\mathrm{P}_{\chi}\left(r_{I C}\right)=8 \pi^{2} \xi_{O 0} G \rho_{0}^{2} \beta \frac{r_{I C}^{2} L_{\rho}^{2}}{\mathrm{f}_{\mathrm{C}}\left(\frac{r_{O C}}{L_{\rho}}, 0\right)}\left[\mathrm{f}_{\chi}\left(\frac{r_{O C}}{L_{\rho}}\right)-\mathrm{f}_{\chi}\left(\frac{r_{I C}}{L_{\rho}}\right)\right]
$$

with

$$
\mathrm{f}_{\chi}(x)=x^{3}\left[-\frac{r_{I C}^{2}}{3 L_{\rho}^{2}}+\frac{1}{5}\left(1+\frac{r_{I C}^{2}}{L_{\rho}^{2}}\right) x^{2}-\frac{13}{70} x^{4}\right] .
$$

Only terms larger than $r_{O C}^{4} / L_{\rho}^{4}$ have been retained. Note that because $r_{I C} / r_{O C} \leq 0.35$ for the Earth, many terms can be neglected that contain combinations of $r_{I C}$ and $r_{O C}$, like $r_{I C}^{4} /\left(r_{O C}^{2} L_{\rho}^{2}\right)$ or $r_{I C}^{5} / r_{O C}^{5}$. Note also that equation (25) of Labrosse (2003) is recovered by neglecting all terms of orders higher than 2 in $r_{O C} / L_{\rho}$, even though the present expression is obtained as a compositional energy while that in Labrosse (2003) was computed as a gravitational energy. This correspondence was already discussed by Braginsky and Roberts (1995) and found valid only for a constant $\beta$.

The total compositional energy extracted since the onset of inner core crystallisation, $\mathcal{X}$, is computed by directly integrating $P_{\chi}$ (eq. A.14), which poses no difficulty since it is a polynomial of $r_{I C}$. This gives

$$
\mathcal{X}=\frac{8 \pi^{2}}{15} \xi_{O 0} G \rho_{0}^{2} \beta \frac{r_{I C}^{3} r_{O C}^{5}}{L_{\rho}^{3} \mathrm{f}_{\mathrm{C}}\left(\frac{r_{O C}}{L_{\rho}}, 0\right)}\left[1-\frac{r_{I C}^{2}}{r_{O C}^{2}}+\frac{3 r_{I C}^{2}}{5 L_{\rho}^{2}}-\frac{13 r_{O C}^{2}}{14 L_{\rho}^{2}}+\frac{5}{18} \frac{r_{I C}^{3} L_{\rho}^{2}}{r_{O C}^{5}}\right] .
$$

This expression gives a numerical value less than $2 \%$ different from the one computed using the full expression (eq. A.13).

\section{Appendix A.5. Radiogenic heating}

The entropy contribution from radiogenic heating writes

$$
\begin{aligned}
S_{R} & =\frac{4 \pi \rho_{0} L_{\rho}^{3} h(t)}{T_{L}\left(r_{I C}\right)}\left(1-\frac{r_{I C}^{2}}{L_{\rho}^{2}}-A_{\rho} \frac{r_{I C}^{4}}{L_{\rho}^{4}}\right)^{-\gamma} \int_{r_{I C} / L_{\rho}}^{r_{O C} / L_{\rho}}\left(1-u^{2}-A_{\rho} u^{4}\right)^{1-\gamma} u^{2} \mathrm{~d} u, \\
& =\frac{4 \pi \rho_{0} L_{\rho}^{3} h(t)}{3 T_{L}\left(r_{I C}\right)}\left(1-\frac{r_{I C}^{2}}{L_{\rho}^{2}}-A_{\rho} \frac{r_{I C}^{4}}{L_{\rho}^{4}}\right)^{-\gamma}\left[\mathrm{f}_{\mathrm{C}}\left(\frac{r_{O C}}{L_{\rho}},-\gamma\right)-\mathrm{f}_{\mathrm{C}}\left(\frac{r_{I C}}{L_{\rho}},-\gamma\right)\right] .
\end{aligned}
$$

Together with equation (A.4), this gives for the temperature at which radiogenic dissipation occurs

$$
T_{R}=T_{L}\left(r_{I C}\right)\left(1-\frac{r_{I C}^{2}}{L_{\rho}^{2}}-A_{\rho} \frac{r_{I C}^{4}}{L_{\rho}^{4}}\right)^{\gamma} \frac{\mathrm{f}_{\mathrm{C}}\left(\frac{r_{O C}}{L_{\rho}}, 0\right)-\mathrm{f}_{\mathrm{C}}\left(\frac{r_{I C}}{L_{\rho}}, 0\right)}{\mathrm{f}_{\mathrm{C}}\left(\frac{r_{O C}}{L_{\rho}},-\gamma\right)-\mathrm{f}_{\mathrm{C}}\left(\frac{r_{I C}}{L_{\rho}},-\gamma\right)} .
$$




\section{Appendix A.6. Entropy production by conduction along the isentrope}

The contribution of conduction along the isentropic gradient to the entropy balance is computed from its definition (eq. 32), the definition of the radial dependence of conductivity (eq. 20) and the expression of the isentropic temperature profile (eq.11) which gives

$$
\begin{aligned}
S_{k} & =16 \pi \gamma^{2} k_{0} L_{\rho} \int_{r_{I C} / L_{\rho}}^{r_{O C} / L_{\rho}}\left(1-A_{k} u^{2}\right)\left(\frac{1+2 A_{\rho} u^{2}}{1-u^{2}-A_{\rho} u^{4}}\right)^{2} u^{4} \mathrm{~d} u \\
& =16 \pi \gamma^{2} k_{0} L_{\rho}\left[f_{k}\left(\frac{r_{O C}}{L_{\rho}}\right)-f_{k}\left(\frac{r_{I C}}{L_{\rho}}\right)\right]
\end{aligned}
$$

with

$$
\begin{aligned}
f_{k}(x)= & 4\left(1+\frac{A_{k}}{A_{\rho}}\right) x-\frac{4}{3} A_{k} x^{3}+\frac{x\left[A_{k}+2 A_{\rho}-\left(A_{k}+A_{\rho}+2 A_{k} A_{\rho}\right) x^{2}\right]}{2 A_{\rho}\left(1-x^{2}-A_{\rho} x^{4}\right)} \\
& +\frac{A_{\rho}\left(7+20 A_{\rho}-7 \sqrt{1+4 A_{\rho}}\right)+A_{k}\left(9+32 A_{\rho}-\left(9+14 A_{\rho}\right) \sqrt{1+4 A_{\rho}}\right)}{4 A_{\rho}^{3 / 2} \sqrt{\frac{1}{2}+2 A_{\rho}} \sqrt{1-\sqrt{1+4 A_{\rho}}}} \arctan \left(\frac{\sqrt{2 A_{\rho}} x}{\sqrt{1-\sqrt{1+4 A_{\rho}}}}\right) \\
& -\frac{A_{\rho}\left(7+20 A_{\rho}+7 \sqrt{1+4 A_{\rho}}\right)+A_{k}\left(9+32 A_{\rho}+\left(9+14 A_{\rho}\right) \sqrt{1+4 A_{\rho}}\right.}{4 A_{\rho}^{3 / 2} \sqrt{\frac{1}{2}+2 A_{\rho}} \sqrt{1+\sqrt{1+4 A_{\rho}}}} \arctan \left(\frac{\sqrt{2 A_{\rho}} x}{\sqrt{1+\sqrt{1+4 A_{\rho}}}}\right) .
\end{aligned}
$$

A development of this function to the same order as for the other ones can also be used:

$$
f_{k}(x)=\frac{x^{5}}{5}\left[1+\frac{5}{7}\left(2-A_{k}+4 A_{\rho}\right) x^{2}+\frac{5}{9}\left(3+10 A_{\rho}+4 A_{\rho}^{2}-2 A_{k}\left(1+2 A_{\rho}\right)\right) x^{4}+O[x]^{5}\right] .
$$

\section{Appendix A.7. Dissipation temperature and radioactivity}

The effective dissipation temperature, $T_{\Phi}$, is computed assuming a uniform distribution in the core (eq. 36), which gives

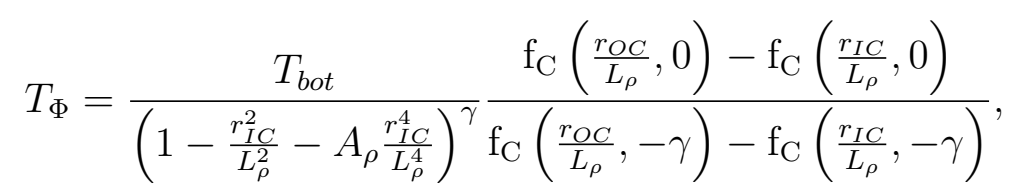

$T_{\text {bot }}$ being the bottom temperature, ie $T_{L}\left(r_{I C}\right)$ when there is an inner core or $T_{c}$ before that time. Since I assume that radioactive elements are always uniformly distributed in the core, the expression for $T_{R}$ is exactly the same: $T_{R}=T_{\Phi}$.

\section{References}

Abe, Y., 1997. Thermal and chemical evolution of the terrestrial magma ocean. Phys. Earth Planet. Inter. 100, 27-39.

Alfè, D., Gillan, M. J., Price, G. D., 1999. The melting curve of iron at the pressures of the

Earth's core from ab initio calculations. Nature 401, 462-463. 
Alfè, D., Gillan, M. J., Price, G. D., 2001. Thermodynamics of hexagonal-close-packed iron under Earth's core conditions. Phys. Rev. B 64, 045123.

Alfè, D., Gillan, M. J., Price, G. D., 2002. Composition and temperature of the Earth's core constrained by combining ab initio calculations and seismic data. Earth Planet. Sci. Lett. 195, 91-98.

Alfè, D., Gillan, M. J., Price, G. D., 2007. Temperature and composition of the Earth's core. Contemporary Physics 48, 63-80.

Andrault, D., Bolfan-Casanova, N., Nigro, G. L., Bouhifd, M. A., Garbarino, G., Mezouar, M., Apr. 2011. Solidus and liquidus profiles of chondritic mantle: Implication for melting of the Earth across its history. Earth Planet. Sci. Lett. 304, 251-259.

Andrault, D., Pesce, G., Bouhifd, M. A., Bolfan-Casanova, N., Hénot, J.-M., Mezouar, M., 2014. Melting of subducted basalt at the core-mantle boundary. Science 344, 892-895.

Aubert, J., Amit, H., Hulot, G., 2007. Detecting thermal boundary control in surface flows from numerical dynamos. Phys. Earth Planet. Inter. 160, 143-156, doi: 10.1016/j.pepi.2006.11.003.

Aubert, J., Labrosse, S., Poitou, C., Jan 2009. Modelling the palaeo-evolution of the geodynamo. Geophys. J. Int. 179, 1414-1428.

Backus, G. E., 1975. Gross thermodynamics of heat engines in deep interior of Earth. Proc. Nat. Acad. Sci. U.S.A. 72, 1555-1558.

Becker, T. W., Conrad, C. P., Buffett, B. A., Müller, R. D., 2009. Past and present seafloor age distributions and the temporal evolution of plate tectonic heat transport. Earth Planet. Sci. Lett. 278, 233-242.

Bercovici, D., Ricard, Y. R., 2014. Plate tectonics, damage and inheritance. Nature 508, 513516.

Bouhifd, M. A., Andrault, D., Bolfan-Casanova, N., Hammouda, T., Devidal, J. L., 2013. Metal-silicate partitioning of $\mathrm{Pb}$ and $\mathrm{U}$ : Effects of metal composition and oxygen fugacity. Geochim. Cosmochim. Acta 114, 13-28.

Braginsky, S. I., Roberts, P. H., 1995. Equations governing convection in Earth's core and the geodynamo. Geophys. Astrophys. Fluid Dyn 79, 1-97.

Braginsky, S. J., 1963. Structure of the F layer and reasons for convection in the Earth's core. Doklady Akad. Nauk SSSR 149, 8-10.

Brummell, N. H., Clune, T. L., Toomre, J., 2002. Penetration and Overshooting in Turbulent Compressible Convection. Astrophys. J. 570, 825-854.

Buffett, B., 2007. 8.12 - core-mantle interactions. In: Schubert, G. (Ed.), Treatise on Geophysics. Elsevier, Amsterdam, pp. 345 - 358.

Buffett, B. A., Garnero, E. J., Jeanloz, R., 2000. Sediments at the top of Earth's core. Science 290, 1338-1342.

Buffett, B. A., Huppert, H. E., Lister, J. R., Woods, A. W., 1992. Analytical model for solidification of the Earth's core. Nature 356, 329-331. 
Buffett, B. A., Huppert, H. E., Lister, J. R., Woods, A. W., 1996. On the thermal evolution of the Earth's core. J. Geophys. Res. 101, 7989-8006.

Cao, A., Romanowicz, B., 2004. Constraints on density and shear velocity contrasts at the inner core boundary. Geophys. J. Int. 157, 1146-1151.

Cébron, D., Maubert, P., Le Bars, M., 2010. Tidal instability in a rotating and differentially heated ellipsoidal shell. Geophys. J. Int. 182, 1311-1318.

Christensen, U. R., 2010. Dynamo scaling laws and applications to the planets. Space Science Reviews 152, 565-590.

Christensen, U. R., Aubert, J., 2006. Scaling properties of convection-driven dynamos in rotating spherical shells and application to planetary magnetic fields. Geophys. J. Int. 166, 97-114.

Coltice, N., Rolf, T., Tackley, P. J., Labrosse, S., 2012. Dynamic causes of the relation between area and age of the ocean floor. Science 336, 335-338.

de Koker, N., Steinle-Neumann, G., Vlček, V., 2012. Electrical resistivity and thermal conductivity of liquid Fe alloys at high $\mathrm{P}$ and $\mathrm{T}$, and heat flux in Earth's core. Proc. Nat. Acad. Sci. U.S.A. 109, 4070-4073.

de Wijs, G. A., Kresse, G., Vocadlo, L., Dobson, D., Alfe, D., Gillan, M. J., Price, G. D., 1998. The viscosity of liquid iron at the physical conditions of the Earth's core. Nature 392, 805-807.

Dziewonski, A. M., Anderson, D. L., 1981. Preliminary reference Earth model. Phys. Earth Planet. Inter. 25, 297-356.

Fiquet, G., Auzende, A. L., Siebert, J., Corgne, A., Bureau, H., Ozawa, H., Garbarino, G., 2010. Melting of peridotite to 140 GigaPascals. Science 329, 1516-1518.

Garnero, E. J., Helmberger, D. V., Grand, S. P., 1993. Constraining outermost core velocity with SmKS waves. Geophysical Research Letters 20, 2463-2466.

Gomi, H., Ohta, K., Hirose, K., Labrosse, S., Caracas, R., Verstraete, M. J., Hernlund, J. W., 2013. The high conductivity of iron and thermal evolution of the Earth's core. Phys. Earth Planet. Inter. 224, 88-103.

Grigné, C., Labrosse, S., 2001. Effects of continents on Earth cooling: Thermal blanketing and depletion in radioactive elements. Geophys. Res. Lett. 28, 2707-2710.

Gubbins, D., 1977. Energetics of the Earth's core. J. Geophys. 43, 453-464.

Gubbins, D., 2007. Geomagnetic constraints on stratification at the top of Earth's core. Earth Planets Space 59, 661-664.

Gubbins, D., Alfè, D., Davies, C., Pozzo, M., 2015. On core convection and the geodynamo: Effects of high electrical and thermal conductivity. Phys. Earth Planet. Inter. this issue.

Gubbins, D., Alfè, D., Davies, C. J., 2013. Compositional instability of Earth's solid inner core. Geophys. Res. Lett. 40, 1084-1088. 
Gubbins, D., Alfè, D., Masters, G., Price, G. D., Gillan, M. J., 2003. Can the Earth's dynamo run on heat alone? Geophys. J. Int. 155, 609-622.

Gubbins, D., Alfè, D., Masters, G., Price, G. D., Gillan, M. J., 2004. Gross thermodynamics of 2-component core convection. Geophys. J. Int. 157, 1407-1414.

Gubbins, D., Masters, T. G., Jacobs, J. A., 1979. Thermal evolution of the Earth's core. Geophys. J. R. Astr. Soc. 59, 57-99.

Gubbins, D., Thomson, C. J., Whaler, K. A., 1982. Stable regions in the Earth's liquid core. Geophys. J. R. Astr. Soc. 68, 241-251.

Gunnarsson, O., Calandra, M., Han, J. E., Oct 2003. Colloquium : Saturation of electrical resistivity. Rev. Mod. Phys. 75, 1085-1099.

Helffrich, G., Kaneshima, S., 2010. Outer-core compositional stratification from observed core wave speed profiles. Nature 468, 807-810.

Hernlund, J. W., Labrosse, S., 2007. Geophysically consistent values of the perovskite to post-perovskite transition Clapeyron slope. Geophys. Res. Lett. 34, L05309, doi:10.1029/2006GL028961.

Hirose, K., Labrosse, S., Hernlund, J. W., 2013. Composition and state of the core. Ann. Rev. Earth Planet. Sci. 41, 657-691.

Hulot, G., Eymin, C., Langlais, B., Mandea, M., Olsen, N., 2002. Small-scale structure of the geodynamo inferred from Oersted and Magsat satellite data and magsat satellite data. Nature 416, 620-623.

Ichikawa, H., Tsuchiya, T., Tange, Y., 2014. The PVT equation of state and thermodynamic properties of liquid iron. J. Geophys. Res. 119, 240-252.

Jaupart, C., Labrosse, S., Mareschal, J.-C., 2007. 7.06 - temperatures, heat and energy in the mantle of the earth. In: Schubert, G. (Ed.), Treatise on Geophysics. Elsevier, Amsterdam, pp. $253-303$.

Koper, K. D., Dombrovskaya, M., 2005. Seismic properties of the inner core boundary from PKiKP/P amplitude ratios. Earth Planet. Sci. Lett. 237, 680-694.

Labrosse, S., 2002. Hotspots, mantle plumes and core heat loss. Earth Planet. Sci. Lett. 199, $147-156$.

Labrosse, S., 2003. Thermal and magnetic evolution of the Earth's core. Phys. Earth Planet. Inter. 140, 127-143.

Labrosse, S., 2014. Thermal and compositional stratification of the inner core. C. R. Geosciences 346, 119-129.

Labrosse, S., Hernlund, J. W., Coltice, N., 2007. A crystallizing dense magma ocean at the base of Earth's mantle. Nature 450, 866-869.

Labrosse, S., Jaupart, C., 2007. Thermal evolution of the Earth: Secular changes and fluctuations of plate characteristics. Earth Planet. Sci. Lett. 260, 465-481. 
Labrosse, S., Macouin, M., 2003. The inner core and the geodynamo. C. R. Geosciences 335 (1), $37-50$.

Labrosse, S., Poirier, J.-P., Le Mouël, J.-L., 1997. On cooling of the Earth's core. Phys. Earth Planet. Inter. 99, 1-17.

Labrosse, S., Poirier, J.-P., Le Mouël, J.-L., 2001. The age of the inner core. Earth Planet. Sci. Lett. 190, 111-123.

Laio, A., Bernard, S., Chiarotti, G. L., Scandolo, S., Tosatti, E., 2000. Physics of iron at Earth's core conditions. Science 287, 1027-1030.

Lay, T., Hernlund, J., Buffett, B. A., 2008. Core-mantle boundary heat flow. Nature Geosci. 1, $25-32$.

Le Bars, M., Cébron, D., Le Gal, P., 2015. Flows driven by libration, precession, and tides. Ann. Rev. Fluid Mech. 47, 163-193.

Lister, J. R., 2003. Expressions for the dissipation driven by convection in the Earth's core. Phys. Earth Planet. Inter. 140, 145-158.

Lister, J. R., Buffett, B. A., 1995. The strength and efficiency of the thermal and compositional convection in the geodynamo. Phys. Earth Planet. Inter. 91, 17-30.

Lister, J. R., Buffett, B. A., 1998. Stratification of the outer core at the core-mantle boundary. Phys. Earth Planet. Inter. 105, 5-19.

Loper, D. E., 1978. The gravitationally powered dynamo. Geophys. J. R. Astr. Soc. 54, 389-404.

Loyd, S. J., Becker, T. W., Conrad, C. P., Lithgow-Bertellonifi, C., Corsetti, F. A., 2007. Time variability in cenozoic reconstructions of mantle heat flow: Plate tectonic cycles and implications for Earth's thermal evolution. Proc. Natl. Acad. Sci. USA 104, 14266-14271.

Malavergne, V., Tarrida, M., Combes, R., Bureau, H., Jones, J., Schwandt, C., 2007. New high-pressure and high-temperature metal/silicate partitioning of $\mathrm{U}$ and $\mathrm{Pb}$ : Implications for the cores of the Earth and Mars. Geochim. Cosmochim. Acta 71, 2637-2655.

Masters, G., Gubbins, D., 2003. On the resolution of density within the Earth. Phys. Earth Planet. Inter. 140, 159-167.

McDonough, W. F., Dec. 2003. Compositional Model for the Earth's Core. Treatise on Geochemistry 2, 547-568.

Mollett, S., 1984. Thermal and magnetic constraints on the cooling of the Earth. Geophys. J. R. Astr. Soc. 76, 653-666.

Mosenfelder, J., Asimow, P., Ahrens, T., 2007. Thermodynamic properties of $\mathrm{Mg}_{2} \mathrm{SiO}_{4}$ liquid at ultra-high pressures for shock measurements to $200 \mathrm{GPa}$ on forsterite and wadsleyite. J. Geophys. Res. 112, B06208.

Nakagawa, T., Tackley, P. J., 2010. Influence of initial CMB temperature and other parameters on the thermal evolution of Earth's core resulting from thermochemical spherical mantle convection. Geochem. Geophys. Geosys. 11, Q06001. 
Nimmo, F., 2002. Why does Venus lack a magnetic field? Geology 30, 987-990.

Nimmo, F., 2007. Treatise on Geophysics. Vol. 8. Elsevier, Ch. 2. Energetics of the core, pp. 253-303.

Nimmo, F., Price, G. D., Brodholt, J., Gubbins, D., 2004. The influence of potassium on core and geodynamo evolution. Geophys. J. Int. 156, 263-376.

Nomura, R., Hirose, K., 2014. Metal-silicate partitioning of lithophile elements up to 138 GPa and $5500 \mathrm{~K}$ and implications for potassium and light elements in the core. Geochim. Cosmochim. ActaSubmitted.

Ohta, K., Kuwayama, Y., Shimizu, K., Yagi, T., Hirose, K., Ohishi, Y., 2014. Measurements of electrical and thermal conductivity of iron under earth's core conditions. AGU Fall Meeting Abstracts.

Ozawa, H., Hirose, K., Mitome, M., Bando, Y., Sata, N., Ohishi, Y., 2009. Experimental study of reaction between perovskite and molten iron to $146 \mathrm{GPa}$ and implications for chemically distinct buoyant layer at the top of the core. Phys. Chem. Minerals 36, 355-363.

Ozima, M., Seki, K., Terada, N., Miura, Y. N., Podosek, F. A., Shinagawa, H., 2005. Terrestrial nitrogen and noble gases in lunar soils. Nature 436, 655-659.

Perrillat, J.-P., Mezouar, M., Garbarino, G., Bauchau, S., 2010. In situ viscometry of highpressure melts in the Paris-Edinburgh cell: application to liquid FeS. High Pressure Research 30, 415-423.

Poirier, J.-P., 1988. Transport properties of liquid metals and viscosity of the Earth's core. Phys. Earth Planet. Inter. 92, 99-105.

Poirier, J.-P., 1994. Light elements in the Earth's core: A critical review. Phys. Earth Planet. Inter. 85, 319-337.

Poirier, J.-P., 2000. Introduction to the physics of the Earth's interior, 2nd Edition. Cambridge University Press.

Pozzo, M., Davies, C., Gubbins, D., Alfè, D., 2014. Thermal and electrical conductivity of solid iron and iron-silicon mixtures at Earth's core conditions. Earth and Planetary Science Letters 393, 159-164.

Pozzo, M., Davies, C. J., Gubbins, D., Alfè, D., 2012. Thermal and electrical conductivity of iron at Earth's core conditions. Nature 485, 355-358.

Rama Murthy, V., van Westrenen, W., Fei, Y., 2003. Radioactive heat sources in planetary cores: Experimental evidence for potassium. Nature 423, 163-165.

Ricard, Y., 2007. Treatise of Geophysics. Mantle dynamics. Vol. 7. Elsevier, Ch. Physics of mantle convection, pp. 253-303.

Roberts, P. H., Jones, C. A., Calderwood, A. R., 2003. Energy fluxes and ohmic dissipation in the Earth's core. In: Jones, C. A., Soward, A. M., Zhang, K. (Eds.), Earth's Core and Lower Mantle. Taylor \& Francis, London, pp. 100-129. 
Rost, S., Garnero, E. J., Williams, Q., Manga, M., 2005. Seismological constraints on a possible plume root at the core-mantle boundary. Nature 435, 666-669, doi: 10.1038/nature03620.

Seagle, C. T., Cottrell, E., Fei, Y., Hummer, D. R., Prakapenka, V. B., 2013. Electrical and thermal transport properties of iron and iron-silicon alloy at high pressure. Geophys. Res. Lett. 40, 5377-5381.

Sleep, N. H., 2000. Evolution of the mode of convection within terrestrial planets. J. Geophys. Res. 105, 17563-17578.

Song, X., Helmberger, D. V., 1995. A P wave velocity model of Earth's core. J. Geophys. Res. 100, 9817-9830.

Souriau, A., Poupinet, G., 1991. The velocity profile at the base of the liquid core from PKP(BC+Cdiff) data: An argument in favour of radial inhomogeneity. Geophys. Res. Lett. 18, 2023-2026.

Stacey, F. D., Loper, D. E., 1984. Thermal histories of the core and mantle. Phys. Earth Planet. Inter. 36, 99-115.

Stevenson, D., Spohn, T., Schubert, G., 1983. Magnetism and thermal evolution of the terrestrial planets. Icarus 54, 466-489.

Stevenson, D. J., 2007. Core exsolution: A likely consequence of giant impacts and a likely energy source for the geodynamo. Fall Meet. Suppl., Abstract U21D-02.

Stixrude, L., de Koker, N., Sun, N., Mookherjee, M., Karki, B. B., 2009. Thermodynamics of silicate liquids in the deep Earth. Earth Planet. Sci. Lett. 278, 226-232.

Tackley, P. J., 2000. Mantle convection and plate tectonics: Toward an integrated physical and chemical theory. Science 288, 2002-2007.

Tanaka, S., 2007. Possibility of a low P-wave velocity layer in the outermost core from global SmKS waveforms. Earth Planet. Sci. Lett. 259, 486-499.

Tanaka, S., Hamaguchi, H., 1993. Velocities and chemical stratification in the outermost core. J. Geomag. Geoelecr. 45, 1287-1301.

Tarduno, J. A., Cottrell, R. D., Watkeys, M. K., Hofmann, A., Doubrovine, P. V., Mamajek, E. E., Liu, D., Sibeck, D. G., Neukirch, L. P., Usui, Y., 2010. Geodynamo, solar wind, and magnetopause 3.4 to 3.45 billion years ago. Science 327, 1238-1240.

Thomas, C. W., Liu, Q., Agee, C. B., Asimow, P. D., Lange, R. A., 2012. Multi-technique equation of state for $\mathrm{Fe}_{2} \mathrm{SiO}_{4}$ melt and the density of Fe-bearing silicate melts from 0 to 161 GPa. J. Geophys. Res. 117, B10206.

Tilgner, A., 2005. Precession driven dynamos. Phys. Fluids 17, 034104.

Usui, Y., Tarduno, J. A., Watkeys, M., Hofmann, A., Cottrell, R. D., 2009. Evidence for a 3.45billion-year-old magnetic remanence: Hints of an ancient geodynamo from conglomerates of South Africa. Geochem. Geophys. Geosyst. 10, Q09Z07.

Vočadlo, L., Alfè, D., Gillan, M. J., Price, G. D., 2003. The properties of iron under core conditions from first principles calculations. Phys. Earth Planet. Inter. 140, 101-125. 
Whaler, K. A., 1980. Does the whole core convect? Nature 287, 528-530.

Wheeler, K. T., Walker, D., Fei, Y. W., Minarik, W. G., McDonough, W. F., 2006. Experimental partitioning of uranium between liquid iron sulfide and liquid silicate: Implications for radioactivity in the Earth's core. Geochim. Cosmochim. Acta 70, 1537-1547.

Williams, Q., Garnero, E. J., 1996. Seismic evidence for partial melt at the base of the Earth's mantle. Science 273, 1528.

Williamson, E. D., Adams, L. H., 1923. Density distribution in the Earth. J. Washington Acad. Sci. 13, 413-428.

Zahn, J.-P., 1991. Convective penetration in stellar interiors. Astron. Astrophys. 252, 179-188.

Zhang, P., Cohen, R. E., Haule, K., 2015. Effects of electron correlations on transport properties of iron at Earth's core conditions. Nature 517, 605-607. 